\title{
Channel Characterization of IRS-based Visible Light Communication Systems
}

\author{
Amr M. Abdelhady, Osama Amin, Ahmed K. Sultan, and Mohamed-Slim \\ Alouini, and Basem Shihada
}

\begin{abstract}
This paper studies the temporal characteristics of the intelligent reflecting surface (IRS)-based visible light communication (VLC) channel using radiometric concepts. Throughout this study, we account for the delays experienced by the transmitted power along the continuum of paths originating at the source, passing through the IRS, reaching the detector. Then, we derive the impulse response of multi-element phase-tunable metasurface and orientation-tunable mirror array-based reflector setups for a general setting of source, reflector, and detector dimensions and relative positions. In addition, we derive simpler expressions for the two special cases, namely, the point source and the large-source small-reflector. Moreover, we present the exact expression for the delay spread and derive lower, upper bounds and asymptotic expressions when the number of reflecting elements increases for both reflector types. Finally, we study the impact of several system parameters on the temporal characterization of the two IRS-based VLC systems.
\end{abstract}

\section{Index Terms}

Intelligent reflecting surfaces, Metasurfaces, Mirror arrays, Visible Light Communications, Channel modeling

\section{INTRODUCTION}

Visible light communication (VLC) offers a viable competitive solution compared with the radio-frequency technology thanks to the spectrum abundance, physical security, and low electromagnetic interference, in addition to the recent wide adoption of light-emitting-diodes for

A. M. Abdelhady, O. Amin, A. K. Sultan, M.-S. Alouini, and B. Shihada are with with the Computer Electrical, and Mathematical Science and Engineering (CEMSE) Division, King Abdullah University of Science and Technology (KAUST), Thuwal, Makkah Province, Kingdom of Saudi Arabia, E-mail: \{amr.abdelhady, osama.amin, ahmed.salem, slim.alouini, basem.shihada\}@kaust.edu.sa. 
lighting [1]. Such favored features promote VLC as one of the main enablers of the sixth generation networks [2], [3] required to support unprecedented data rates, a massive number of devices, and a plethora of services [4]-[6]. Nonetheless, VLC systems performance is hindered by some challenges. For instance, the fidelity of VLC links relies heavily on Line-of-Sight (LoS) existence between the transmitter and the receiver. Moreover, VLC systems are required to fulfill many illumination objectives as spatial uniformity of irradiance distribution, and limited variability of instantaneous radiated optical power [7]. Recently, reconfigurable reflecting surfaces are incorporated in VLC systems to increase the probability of having a LoS link between the transmitter and the receiver [8]. In addition, they can aid VLC systems in achieving a better tradeoff between the communication and the illumination quality of service.

Intelligent reflecting surfaces (IRS) are considered and studied to add more degrees of freedom to optical wireless communication systems [8]-[13]. In [9], [10], Najafi et al. investigated the incorporation of smart mirrors and metasurfaces to relax the LoS requirement for freespace optical links. Additionally, in [11], Ajam et al. used the Huygens-Fresnel principle to derive the channel model of the IRS-based FSO link. Moreover, in [14], Wang et al. derived closed-form expressions for the output power density of IRS-assisted FSO systems where beam splitting is taken into consideration. In [12], Valagiannopoulos et al. used the metasurfaces to cover optical transmitters to add a programmable beam directivity feature for the optical communication system. Similarly, in [13], Ndjiongue et al. proposed the use of intelligent surfaces at the photodetector side to improve the transmission range. Recently, we proposed metasurface and mirror array-based VLC systems and proved their effectiveness in focusing the incident power towards the detector in [8]. The superiority offered by IRS-based optical systems over their conventional counterparts is obvious in terms of received power and delay spread for non-LoS scenarios. As for LoS scenarios, a received power gain is achieved when IRS-based VLC systems are adopted whilst increasing the delay spread. The full exploitation of VLC networks necessitates thorough characterization of the optical wireless channel under different deployment scenarios.

Optical wireless channel modeling has been investigated thoroughly in the literature [15]-[18], [19, Ch. 1] using the radiometric theoretical framework. Indoor optical wireless channel modeling encompassed many scenarios ranging from deterministic channel models to stochastic ones [20, Ch 3.1]. The deterministic channel models account for the relative transmitter, receiver positions, and orientations in an empty room; however, the stochastic models account for blockages due 
to furniture and people's motion. The main focus of optical channel modeling is on diffuse reflections (unintentional and designed) as discussed in [20, Ch 3.1] and the references therein. In contrast, less optical channel modeling research used specular reflections resulting from perfect mirrors such as [21]. To account for more advanced reflection characteristics, Rufo et al. proposed employing reflection models based on bidirectional reflectance distribution function as Blinn's or Lafortune's models into the impulse response estimation of the indoor wireless optical channels in [22]. In [23], Miramirkhani et al. compared the widely adopted channel models for the wireless visible light channel and proposed a numerical ray-tracing based channel modeling approach that accommodates general source radiation patterns and mixed specular-diffuse reflections. In the previously mentioned works, the considered reflections were either imposed by the static environment representing the confined indoor space walls or placed deliberately to achieve directed non-line of sight communications. However, the considered reflection design problems featured limited degrees of freedom due to the employed reflectors' static reflection characteristics.

In our previous work [8], we proposed IRS-aided VLC systems and modeled the static frequency flat channel, where the focused power is assumed to reach the detector instantaneously, or equivalently, when the delay spread is negligible compared with the symbol duration. In this work, we explore the temporal dispersive nature of the IRS-based VLC systems imposed by the reflector topology, or the geometric layout of the source, reflector and detector, or the signaling rate where the delay spread can not be neglected compared with the symbol duration. To this end, we first derive the continuous-time channel impulse response and then use it to find the tapped-delay line representation for both metasurface and mirror array-based VLC channels. Moreover, we define the exact delay spread formula and derive a simplified version for the asymptotically large number of elements. Also, we derive an upper bound for the delay spread characterizing the two systems. Finally, we illustrate the effect of different system parameters as the number of reflecting elements, reflector area, relative positions of the source, reflector, and detector on the impulse response, and, consequently, the delay spread for both types of IRS. This enables system designers avoid inter-symbol interference in the considered frequency selective channel, or combat it by designing the required equalizers accordingly. It is worth mentioning that the derived IRS-aided VLC channel model in this work is most suitable for indoor scenarios (residential buildings, hotels, lecture halls, hospitals, to name but a few) imposing strict mobility constraints and consequently the channel can be considered quasi-static [20, Ch. 3.1], [24]. To 
the best of our knowledge, this is the first channel modeling study for IRS-based non-coherent VLC systems.

The rest of this paper is organized as follows: firstly, we present the metasurface and mirror array-based reflectors system models in section II. Then, we derive the continuous channel impulse response in section III. Next, we provide the tapped-delay-line channel model and derive the delay spread of the two reflectors for different scenarios in section IV. After that, we present extensive simulations for the two systems in section V. Finally, we give our conclusions regarding the two reflecting systems in section VI.

Notations: In this paper, we represent a vector by two capitalized bold letters as AB, where it starts from $\mathrm{A}$ and ends at $\mathrm{B}$. $\mathbf{A}$ starts from from origin and ends at $\mathrm{A} . \mathbf{A B}=\left[\mathrm{AB}_{x} \mathrm{AB}_{\mathrm{y}} \mathrm{AB}_{z}\right]^{\mathrm{T}}=$ $\mathbf{B}-\mathbf{A}$, where $\mathrm{AB}_{x}, \mathrm{AB}_{y}$, and $\mathrm{AB}_{z}$ representing its $x, y$ and $z$ coordinates, respectively, and $(.)^{\mathrm{T}}$ represents the transpose operator. $\widehat{\mathbf{A B}}$ is the unit vector of $\mathbf{A B}$ and represents the direction of a vector starting at point $\mathrm{A}$ and ending at point $\mathrm{B}$. $\mathrm{AB}$ represents a line segment between the points A and B. Moreover, we use $J\left(\frac{a, b}{c, d}\right)$ to denote the determinant of the Jacobian matrix $\bar{J}(a, b, c, d)$ associated with mapping $c$ and $d$ variables into $a$ and $b$ variables, where $\bar{J} \triangleq\left[\left[\frac{\partial a}{\partial c} \frac{\partial b}{\partial c}\right]^{T}\left[\frac{\partial a}{\partial d} \frac{\partial b}{\partial d}\right]^{T}\right]$. Also, we use $\boldsymbol{e}_{k}$ denote the $k$-th column of the identity matrix. Furthermore, we use $\|.\|_{2}$ to denote the $\ell_{2}$-norm, while we use $|$.$| to represent the absolute$ value of a scalar. $\mathbb{I}(C)$ represents an indicator function where $\mathbb{I}(C)=1$ if the condition $C$ is satisfied and $\mathbb{I}(C)=0$, otherwise. Regarding the fonts, we use calligraphic and blackboard fonts to represent symbols for sets and matrices, respectively.

\section{System Model}

In this work, we consider the metasurface-based and mirror array -based VLC systems proposed in [8], where a non-coherent light emitting diode (LED) transmitter that is horizontallyoriented and attached to the room ceiling separated vertically by a distance $h_{\mathrm{d}}$ from the detector plane as depicted in Fig. 1 and Fig. 2. The positive $x, y$ and $z$ axes orientations are set such that the $z$-axis is normal to the ceiling, pointing at the room floor, while the $y$-axis is orthogonal to a wall and points inside the room, and the $x$-axis forms a right handed coordinate system with the $y-$ and $z-$ axes. Moreover, we assume an extended planar source spanning an area $A_{\mathrm{s}}=w_{\mathrm{s}} l_{\mathrm{s}}$ where $w_{\mathrm{s}}$ and $l_{\mathrm{s}}$ represent the side lengths of the source rectangular aperture measured along the $x$-direction and $y$-direction, respectively. The transmitter aperture points are characterized by the 
same radiance expressed as [8, Eqn. (1)]

$$
L\left(\theta_{\mathrm{s}}, t\right)=\frac{(m+1) p(t)}{2 \pi A_{\mathrm{s}}} \cos ^{m-1}\left(\theta_{\mathrm{s}}\right),
$$

where $m=-\ln \left(\cos \left(\phi_{1 / 2}\right)\right), \phi_{1 / 2}$ represents the half power beamwidth, $p(t)$ denotes the transmitter instantaneous optical radiated power at time $t, \theta_{\mathrm{s}}$ is the angle between the normal vector exiting the source aperture and the direction of radiance measurement.

Moreover, we assume a receiver having horizontal orientation whose center $x$-direction offset from the source center is $x_{\mathrm{d}}$, while it is offset by $y_{\mathrm{d}}$ from the reflector along the positive $y$ direction, as shown in Fig. 1 and Fig. 2. The detector aperture is assumed to be rectangular with edges parallel to the $x$ and $y$ - axes having side lengths $w_{\mathrm{d}}$ and $l_{\mathrm{d}}$, respectively. Furthermore, the detection pattern is assumed to be Lambertian having a field-of-view of $90^{\circ}$.

Finally, we detail the structure of the considered reflectors in the following sections.

\section{A. Intelligent metasurface reflector}

For the metasurface reflector, we assume a co-planar $n_{\mathrm{p}} \times n_{\mathrm{p}}$ array of identical rectangular optical metasurface patches whose widths and heights are $w_{\mathrm{p}}$ and $h_{\mathrm{p}}$, respectively, lying in a vertical surface orthogonal to the $y$-axis. The offsets between patches along the $z$-axis and the $x$-axis are $\Delta_{h_{\mathrm{p}}}$ and $\Delta_{w_{\mathrm{p}}}$, respectively. The reflector location is defined with respect the source location by a minimum clearance along the positive $z$-direction $z_{\mathrm{s}}$, with a minimum offset $x_{\mathrm{s}}$ along the positive $x$-direction and constant separation $y_{\mathrm{s}}$ along the negative $y$-direction with all offsets being measured from the source center. Moreover, we assume that the introduced phase discontinuity $(\Theta)$ of each metasurface patch is individually controllable and constant along the patch.

\section{B. Intelligent mirror array}

As for the mirror array system, we consider an $n_{\mathrm{M}} \times n_{\mathrm{M}}$ two-dimensional array of identical rectangular mirrors whose centers form a rectangular grid in the $x-z$ plane. We assume that the each mirror supports two rotational degrees of freedom as shown in Fig. 2, such that the mirror orientation is determined by two successive rotations; the first is about the mirror local $z$-axis with an angle $\beta$ and the second is about the rotated version of the mirror local negative $x$-axis (negative $x$-axis) with an angle $\alpha$ as depicted in Fig. 3. In the reflector default position,

$\left(\alpha_{k, \ell}=0 \forall k, \ell, \beta_{k, \ell}=0 \forall k, \ell\right)$, each mirror spans $w_{\mathrm{m}}$ and $h_{\mathrm{m}}$ along the $x$-direction and the 


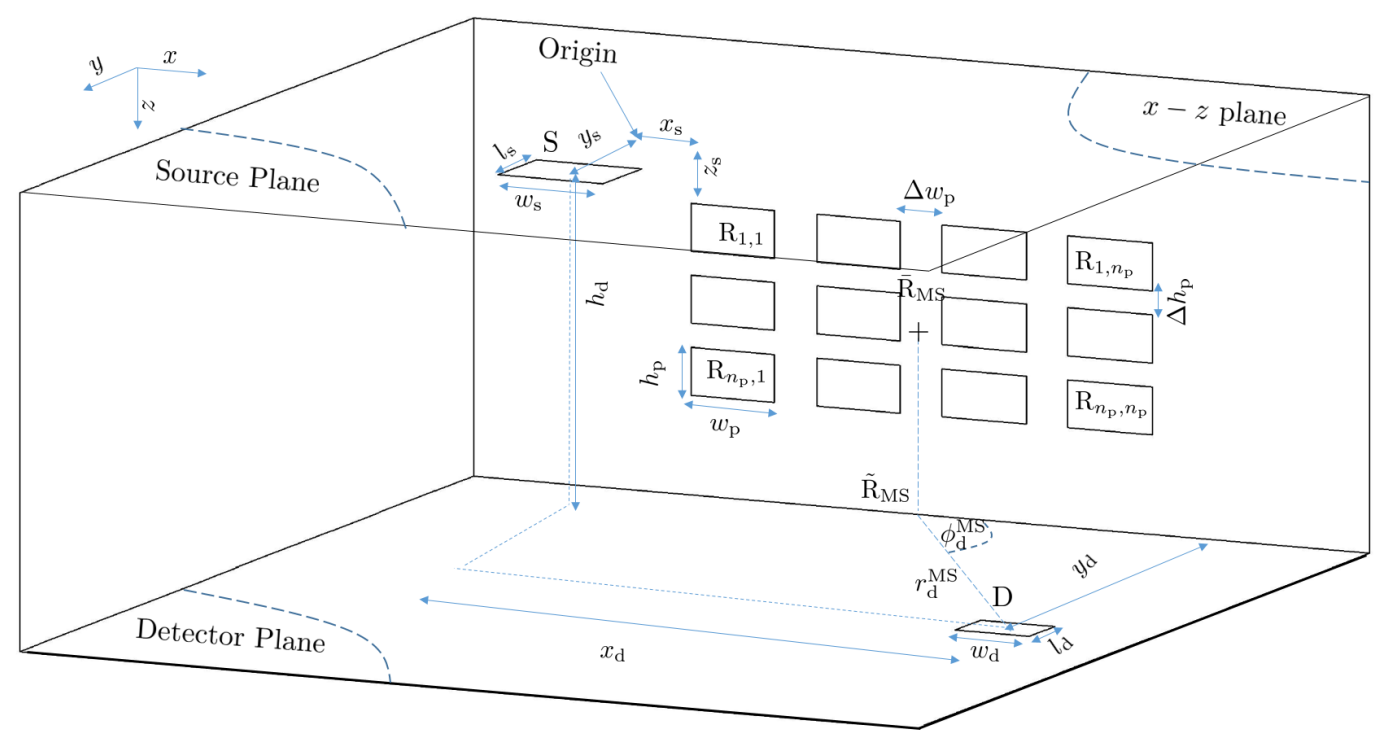

Fig. 1: Metasurface-based IRS Model

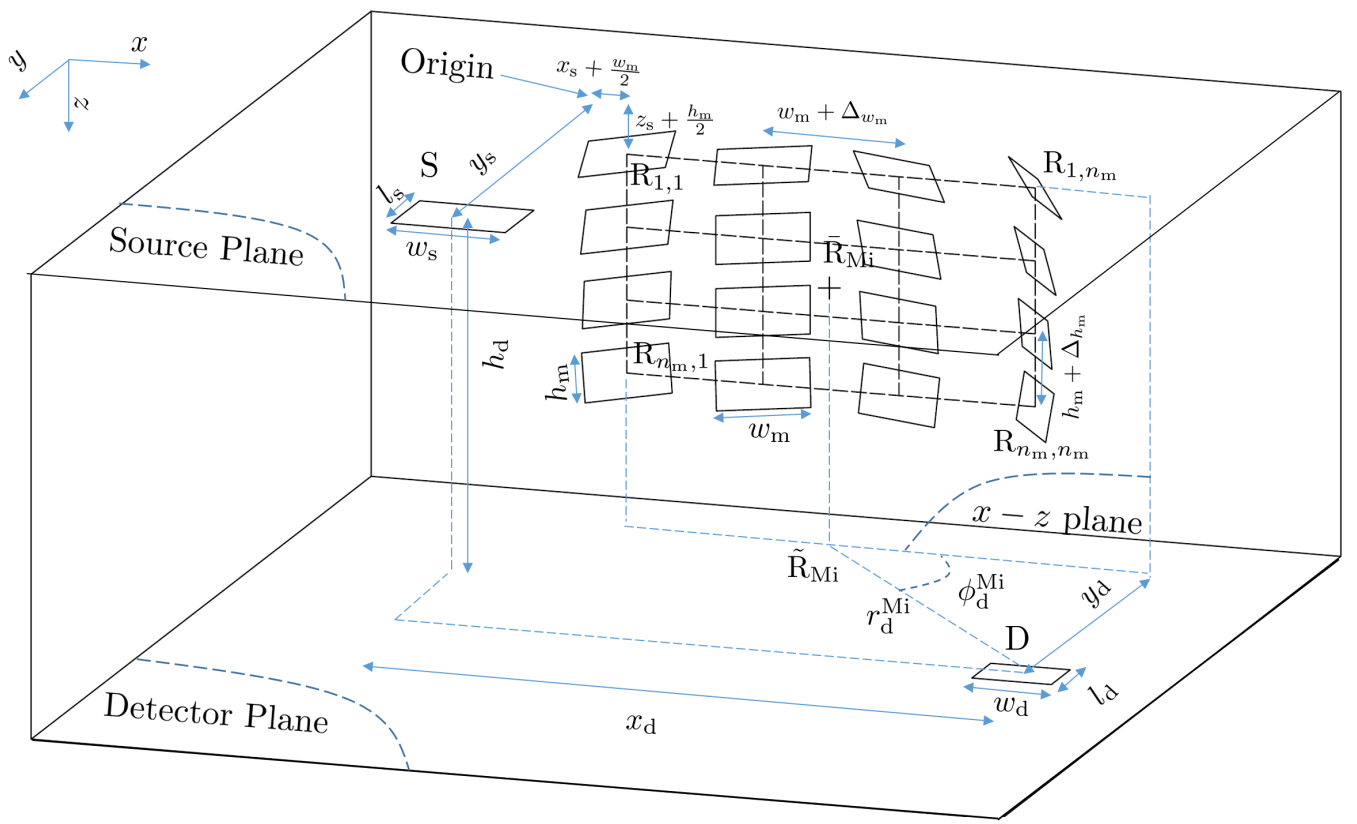

Fig. 2: Mirror Array-based IRS Model

$z$-direction, and adjacent mirrors centers are separated by $w_{\mathrm{m}}+\Delta_{w_{\mathrm{m}}}$ and $h_{\mathrm{m}}+\Delta_{h_{\mathrm{m}}}$ along the $x$-axis and the $z$-axis, respectively.

The thicknesses of the employed materials in the considered reflectors are much larger than the penetration depth in the visible range, and hence, transmittance for both reflector types is 


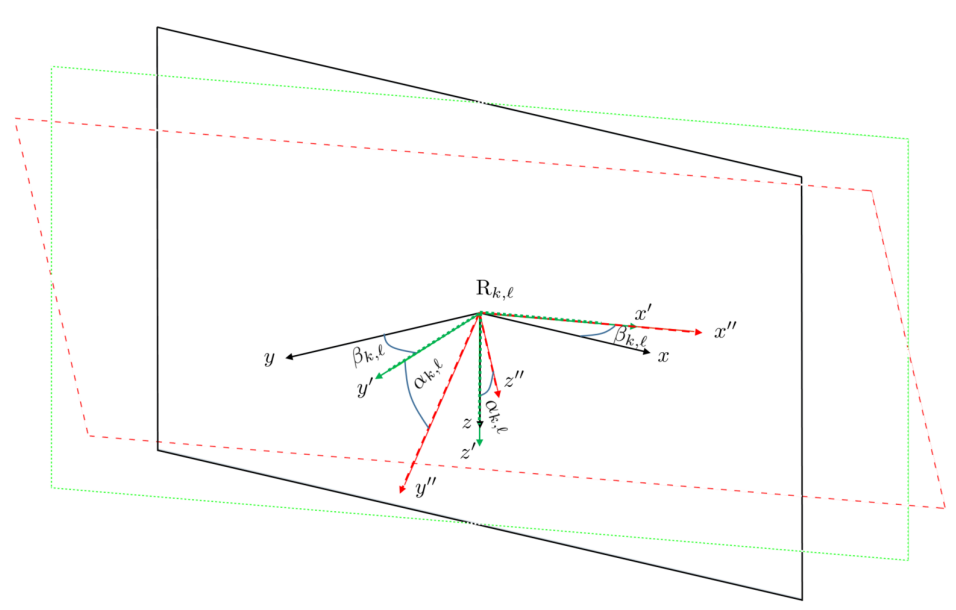

Fig. 3: Rotational Degrees of Freedom of each Mirror

negligible.

\section{Continuous ChANNEL MODEL}

In this section, we derive the impulse response of the two considered IRS-based systems, with the system input being the total radiated optical power and the system output being the received optical power at the detector. Towards this aim, firstly we present the set of assumptions adopted assumptions as follows:

- The phase discontinuities of all metasurface patches are tuned such that the chief incident rays $^{1}$ are reflected at the detector center based on [8, Eqn. 21, 22]. Moreover, they are assumed linear without restrictions on their bounding values.

- The mirrors' orientations are set such that the incident chief rays are reflected at the detector center and determined according to [8, Eqn. 36, 37]. In addition, the angles of rotation $\alpha$ and $\beta$ accept values between $-\pi / 2$ and $\pi / 2$.

- The detector location is perfectly known at the IRS controller and the IRS mirrors orientation or introduced phase discontinuity errors are assumed negligible. ${ }^{2}$

- The surfaces of the both reflectors' elements are perfectly smooth.

\footnotetext{
${ }^{1} \mathrm{~A}$ chief ray is the ray traveling from the source center towards a reflecting element center.

${ }^{2}$ This assumption can be realized in practice by having an incorporated positioning system which reports the detector location IRS-aided VLC system upon significant location variation or periodically based on statistical estimate of the channel coherence time.
} 
- The reflection coefficient magnitude is invariant with the direction of incidence for both reflectors.

- The data transmission is achieved via a monochromatic source. Hence, spectral dependencies of the reflection coefficients can be neglected.

- The detector dimensions are assumed to be small compared with all the distances between the reflector points and the detector points. Hence, irradiance at the detector surface can be assumed constant and equal to that at the detector center.

Before delving into the derivation details, providing a brief discussion on the spatial illumination uniformity and eye safety aspects of the considered setups is due.

Firstly, the spatial illumination uniformity will not be perturbed significantly if the reflected beam is well-focused within the detector area. In such case, only the tails of the power density spatial distribution profile will be outside the detector boundaries which will be insignificant compared with the ambient lighting. The power density profile spatial extent in the detector plane is controlled by the source dimensions and the IRS reflecting elements dimensions [8]. Consequently, a large number of small reflecting elements and a source with small enough physical dimensions should provide the necessary reflected beam confinement.

Secondly, the sensitivity of different parts of the eye depends heavily on the wavelength of the incident light. Eye retina is the most sensitive part to light in the visible frequency range, hence, exposure limits are set with the aim of retinal injury (photoretinitis) avoidance. Towards this end, irradiance at the retina surface is the key quantity to be monitored. The retinal irradiance is proportional to the source radiance [25, Eqn. 3] for the extended source case where the source subtends more than 10 arc minutes measured at the observer. In case of very small angular extent of the source as perceived by the observer (point source), the corneal irradiance represents the determining quantity for setting the exposure limits [26, Ch. 4.5.3]. Eye safety can be guaranteed via pursuing two approaches simultaneously. The first of which is controlling the irradiance at the detector center (focused power density), which represents an upper bound on the corneal irradiance, through controlling the activity of reflecting elements and their reflection coefficients. At the same time, a source with eye safe and adequate radiance for illumination should be chosen (radiance transfer by the reflector arrays will only attenuate it). ${ }^{3}$,

\footnotetext{
${ }^{3}$ Activity refers here to the state of the reflecting element whether it is participating in power focusing or not.
} 
It is worth mentioning that the user data transmission maps to temporal variations in the radiance of the non-coherent LED source. Hence, it is crucial to apply the radiometric principles whilst accounting for the delays of different paths, i.e., all the previously discussed radiometric quantities in [8] are time varying in this study. The adopted radiometric treatment in this work ignores interference as this effect requires special arrangements to be significant when noncoherent sources are employed [27, Ch. 7.3.4]. We denote the radiance measured at a point X, along the direction $\overrightarrow{\mathrm{XY}}$ representing a ray traveling from $\mathrm{X}$ to $\mathrm{Y}$ at a time instant $t$ by $L_{\mathrm{X}, \overrightarrow{\mathrm{XY}}}(t)$. Consequently, the radiance invariance principle in free space can be generalized to account for the temporal dependency as follows:

$L_{\mathrm{X}, \overrightarrow{\mathrm{XY}}}(t)=L_{\mathrm{Y}, \overrightarrow{\mathrm{XY}}}\left(t+\frac{\|\mathrm{XY}\|_{2}}{c}\right)$,

where $c$ is the speed of light in free space.

The irradiance contribution measured at $\mathrm{P}$ of a differential area element $\left(d A_{\mathrm{R}}\right)$ located at an arbitrarily chosen point $\mathrm{R}$ belonging to one of the reflecting elements can be expressed as

$d E_{\mathrm{P}}^{b}(t)=L_{\mathrm{P}, \overrightarrow{\mathrm{RP}}}(t) \cos \left(\theta_{\mathrm{R}, \mathrm{P}}^{b}\right) \cos \left(\theta_{\mathrm{R}}^{\mathrm{P}}\right) B \mathbb{I}\left(\mathrm{I}_{\mathrm{R}, \mathrm{P}}^{b} \in \mathcal{S}\right) d A_{\mathrm{R}} /\|\mathbf{R P}\|_{2}^{2}$,

where $b$, appearing hereafter in the superscripts and the subscripts of the variables indicates the type of the reflector considered, such that $b=\mathrm{Ms}$ and $b=\mathrm{Mi}$ are used for metasurface and mirror array reflectors, respectively. $\theta_{\mathrm{R}, \mathrm{P}}^{b}$, represents the angle between the normal to the differential area element and $\mathrm{RP}, \theta_{\mathrm{R}}^{\mathrm{P}}$ is the angle between the normal to the detector plane and $\mathrm{RP}, B$ is a binary variable that accounts for the inter-element blockage between the reflector array elements, $\mathrm{I}_{\mathrm{R}, \mathrm{P}}^{b}$ is the point in the source plane from which the incident ray on the IRS at $\mathrm{R}$ is reflected towards $\mathrm{P}$, and $\mathcal{S}$ is the set of points representing the source aperture.

Using radiance invariance principle along straight lines, it can be deduced that $L_{\mathrm{P}, \overrightarrow{\mathrm{RP}}}(t)=$ $L_{\mathrm{R}, \overrightarrow{\mathrm{RP}}}\left(t-\frac{\|\mathrm{RP}\|_{2}}{c}\right)$. Based on the specular reflection assumption for both reflector types, it can be deduced that $L_{\mathrm{R}, \overrightarrow{\mathrm{RP}}}(t)=\rho_{\mathrm{b}} L_{\mathrm{R}, \overrightarrow{\mathrm{I}_{\mathrm{R}, \mathrm{P}} \mathrm{R}}}(t)$, where $\rho_{\mathrm{b}}$ represents the reflection efficiency. By exploiting the radiance invariance principle once more, it can be seen clearly that $L \mathrm{R}, \overrightarrow{\mathrm{R}, \mathrm{P}} \mathrm{R}_{\longrightarrow}(t)=$ $L_{\mathrm{I}_{\mathrm{R}, \mathrm{P}}^{b}, \overrightarrow{\mathrm{I}_{\mathrm{R}, \mathrm{P}}^{b} \mathrm{R}}}^{\longrightarrow}\left(t-\frac{\left\|\mathrm{I}_{\mathrm{R}, \mathrm{P}}^{b} \mathrm{R}\right\|_{2}}{c}\right)$. Consequently, $L_{\mathrm{P}, \overrightarrow{\mathrm{RP}}}(t)=\rho_{b} L_{\mathrm{I}_{\mathrm{R}, \mathrm{P}}^{b}, \overrightarrow{\mathrm{I}_{\mathrm{R}, \mathrm{P}}^{b} \mathrm{R}}}\left(t-\frac{\left\|\mathrm{I}_{\mathrm{R}, \mathrm{P}}^{b} \mathrm{R}\right\|_{2}+\|\mathrm{RP}\|_{2}}{c}\right)$.

$\mathrm{By}$ adding the irradiance contributions of all the reflecting elements, the total irradiance at $\mathrm{P}$ can be written as

$E_{\mathrm{P}}^{b}(t)=\rho_{b} \sum_{k, \ell} \iint_{\mathcal{R}_{k, \ell}^{b}} L_{\mathrm{I}_{\mathrm{R}, \mathrm{P}}^{b}} \underset{\mathrm{I}_{\mathrm{R}, \mathrm{P}}^{b} \mathrm{R}}{\longrightarrow}\left(t-\frac{\left\|\mathrm{I}_{\mathrm{R}, \mathrm{P}}^{b} \mathrm{R}\right\|_{2}+\|\mathrm{RP}\|_{2}}{c}\right) \frac{\cos \left(\theta_{\mathrm{R}, \mathrm{P}}^{b}\right) \cos \left(\theta_{\mathrm{R}}^{\mathrm{P}}\right) B \mathbb{I}\left(\mathrm{I}_{\mathrm{R}, \mathrm{P}}^{b} \in \mathcal{S}\right)}{\|\mathbf{R P}\|_{2}^{2}} d A_{\mathrm{R}}$ 
where $\mathcal{R}_{k, l}^{\mathrm{MS}} \forall k, l$ represents the set of points lying on an arbitrarily chosen metasurface patch/ mirror in the $k$-th row and $l$-th column of the reflector array.

By plugging the radiance expression of the uniform-emittance generalized Lambertian source (1), the previous expression can be re-written as

$$
E_{\mathrm{P}}^{b}(t)=\rho_{b} \sum_{k, \ell} \iint_{\mathcal{R}_{k, \ell}^{b}}^{p} \frac{p\left(t-\tau\left(\mathrm{I}_{\mathrm{R}, \mathrm{P}}, \mathrm{R}, \mathrm{P}\right)\right)(m+1) \cos ^{m-1}\left(\theta_{\mathrm{R}, \mathrm{P}}^{\mathrm{I}_{\mathrm{R}}^{b}}\right)}{2 \pi A_{\mathrm{s}}\|\mathbf{R P}\|_{2}^{2}} \cos \left(\theta_{\mathrm{R}, \mathrm{P}}^{b}\right) \cos \left(\theta_{\mathrm{R}}^{\mathrm{P}}\right) B \mathbb{I}\left(\mathrm{I}_{\mathrm{R}, \mathrm{P}}^{b} \in \mathcal{S}\right) d A_{\mathrm{R}},
$$

where $\tau(\mathrm{A}, \mathrm{B}, \mathrm{C})=\frac{\|\mathrm{AB}\|_{2}+\|\mathrm{BC}\|_{2}}{\mathrm{c}}$, and $\theta_{\mathrm{R}, \mathrm{P}}^{\mathrm{I}_{\mathrm{R}}}$ is the angle between the normal to the source aperture and $I_{R, P} R$. Finally the impulse response of the two reflecting systems can be expressed as

$h^{b}(t)=\left.E_{\mathrm{D}}^{b}(t)\right|_{p(t)=\delta(t)} A_{\mathrm{PD}}$,

where $\delta($.$) represents the dirac-delta function such that \delta(0)=\infty, \delta(x)=0 \forall x \neq 0, \int_{-\infty}^{\infty} \delta(x)=$ 1 , and $A_{\mathrm{PD}}=\ell_{\mathrm{d}} w_{\mathrm{d}}$ is the photodetector area.

In the following subsections, we consider special practical cases for the optical source and derive upper bounds or approximations of the corresponding impulse response.

\section{A. Point Source}

We define the optical source to be a "point source" when its dimensions are very small compared with the distances to the reflector and the detector. We first derive the irradiance at the detector center (D) as the source dimensions tend to zero as follows,

$$
\begin{aligned}
E_{\mathrm{D}, \mathrm{PS}}^{b}(t)= & \lim _{w_{\mathrm{s}} \rightarrow 0, \ell_{\mathrm{s}} \rightarrow 0} \rho_{b} \sum_{k, \ell} \iint_{\mathcal{R}_{k, \ell}^{b}} \frac{p\left(t-\tau\left(\mathrm{I}_{\mathrm{R}, \mathrm{P}}^{b}, \mathrm{R}, \mathrm{P}\right)\right)(m-1) \cos ^{m-1}\left(\theta_{\mathrm{R}, \mathrm{P}}^{\mathrm{I}_{\mathrm{R}}^{b}}\right)}{2 \pi A_{\mathrm{s}}\|\mathbf{R D}\|_{2}^{2}} \cos \left(\theta_{\mathrm{R}, \mathrm{P}}^{b}\right) \cos \left(\theta_{\mathrm{R}}^{\mathrm{P}}\right) \\
& \times B \mathbb{I}\left(\mathrm{I}_{\mathrm{R}, \mathrm{P}}^{b} \in \mathcal{S}\right) d A_{\mathrm{R}} .
\end{aligned}
$$

Since $B \leq 1$, and in a similar way to the transition from $[8,(53)]$ to $[8,(54)], E_{\mathrm{D}, \mathrm{PS}}^{b}(t)$ can be upper-bounded as

$$
\begin{aligned}
E_{\mathrm{D}, \mathrm{PS}}^{b}(t) & \leq \rho_{b} \sum_{k, \ell} \frac{p\left(t-\tau\left(\mathrm{S}, \mathrm{R}_{\mathrm{k}, \ell}, \mathrm{D}\right)\right)(m+1) \cos ^{m-1}\left(\theta_{\mathrm{R}_{k, \ell}}^{\mathrm{S}}\right)}{2 \pi\left\|\mathbf{R}_{k, \ell} \mathrm{D}\right\|_{2}^{2}} \cos \left(\theta_{\mathrm{R}_{k, \ell}, \mathrm{D}}^{b}\right) \\
& \times\left.\cos \left(\theta_{\mathrm{R}_{k, \ell}}^{\mathrm{D}}\right) J\left(\frac{\mathrm{I}_{\mathrm{R}, \mathrm{D}, x}^{b}, \mathrm{I}_{\mathrm{R}, \mathrm{D}, y}^{b}}{\mathrm{R}_{x}, \mathrm{R}_{z}}\right)\right|_{\mathrm{R}=\mathrm{R}_{k, \ell}},
\end{aligned}
$$


where $\mathrm{R}_{k, \ell}$ represents the center point of $\mathcal{R}_{k, \ell}, \theta_{\mathrm{R}_{k, \ell}}^{\mathrm{S}}$, represents the angle between the normal to the source aperture and $\mathrm{SR}_{k, \ell}, \theta_{\mathrm{R}_{k, \ell}, \mathrm{D}}^{b}$ represents the angle between the normal to the reflecting element $\mathcal{R}_{k, \ell}$ and $\mathrm{R}_{k, \ell} \mathrm{D}, \theta_{\mathrm{R}_{k, \ell}}^{\mathrm{D}}$ represents the angle between the normal to the photodetector surface and $\mathrm{R}_{k, \ell} \mathrm{D}, \mathrm{I}_{\mathrm{R}, \mathrm{D}, x}^{b}, \mathrm{R}_{x}$, and $\mathrm{R}_{z}$ represent the $x$ - coordinate of $\mathrm{I}_{\mathrm{R}, \mathrm{D}}^{b}$, the $x$ - coordinate of $\mathrm{R}$, and the $z$ - coordinate of $\mathrm{R}$, respectively. Consequently, the impulse response can be expressed as

$$
\begin{aligned}
h_{\mathrm{PS}}^{b}(t) \leq & \rho_{b} \sum_{k, \ell} \frac{A_{\mathrm{PD}}(m+1) \cos ^{m-1}\left(\theta_{\mathrm{R}_{k, \ell}}^{\mathrm{S}}\right)}{2 \pi\left\|\mathbf{R}_{k, \ell} \mathrm{D}\right\|_{2}^{2}} \cos \left(\theta_{\mathrm{R}_{k, \ell}, \mathrm{D}}^{b}\right) \cos \left(\theta_{\mathrm{R}_{k, \ell}}^{\mathrm{D}}\right) \\
& \times\left. J\left(\frac{\mathrm{I}_{\mathrm{R}, \mathrm{D}, x}^{b}, \mathrm{I}_{\mathrm{R}, \mathrm{D}, y}^{b}}{\mathrm{R}_{x}, \mathrm{R}_{z}}\right)\right|_{\mathrm{R}=\mathrm{R}_{k, \ell}} \delta\left(t-\tau\left(\mathrm{S}, \mathrm{R}_{\mathrm{k}, \ell}, \mathrm{D}\right)\right) .
\end{aligned}
$$

For the mirror array reflector, the previous expression can be simplified using $[8,(62)]$ to

$$
h_{\mathrm{PS}}^{\mathrm{Mi}}(t) \leq \rho_{b} \sum_{k, \ell} \frac{A_{\mathrm{PD}}(m+1) \cos ^{m}\left(\theta_{\mathrm{R}_{k, \ell}}^{\mathrm{S}}\right)}{2 \pi\left(\left\|\mathbf{R}_{\boldsymbol{k}, \ell} \mathbf{S}\right\|_{2}+\left\|\mathbf{R}_{\boldsymbol{k}, \ell} \mathrm{D}\right\|_{2}\right)^{2}} \cos \left(\theta_{\mathrm{R}_{k, \ell}}^{\mathrm{D}}\right) \delta\left(t-\tau\left(\mathrm{S}, \mathrm{R}_{\mathrm{k}, \ell}, \mathrm{D}\right)\right) .
$$

As for the metasurface reflector, the impulse response can be simplified by approximating the Jacobian term appearing in (9) in a similar way to $[8,(66)]$, into

$$
h_{\mathrm{PS}}^{\mathrm{Ms}}(t) \approx \rho_{b} \sum_{k, \ell} \frac{A_{\mathrm{PD}}(m+1) \cos ^{m}\left(\theta_{\mathrm{R}_{k, \ell}}^{\mathrm{S}}\right)}{2 \pi\left(\left\|\mathbf{R}_{\boldsymbol{k}, \ell} \mathbf{S}\right\|_{2}+\left\|\mathbf{R}_{\boldsymbol{k}, \ell} \mathbf{D}\right\|_{2}\right)^{2}} \cos \left(\theta_{\mathrm{R}_{k, \ell}}^{\mathrm{D}}\right) \frac{\cos \left(\theta_{\mathrm{R}_{k, \ell} \mathrm{DS}}^{\mathrm{MS}}\right)}{\widehat{\mathbf{N}}_{k, \ell}^{\mathrm{T}} \widehat{\mathbf{R}_{k, \ell} \mathbf{D}}} \delta\left(t-\tau\left(\mathrm{S}, \mathrm{R}_{\mathrm{k}, \ell}, \mathrm{D}\right)\right),
$$

where $\widehat{\mathbf{N}}_{k, \ell}$ represents a unit vector orthogonal to the reflecting element $\mathcal{R}_{k, \ell}$.

\section{B. Large source small reflector}

In this case, we assume the reflector dimensions are very small compared with the minimum distance between source points and reflector points. Similarly, the reflector dimensions are assumed to be very small compared with the minimum distance between reflector points and D. Moreover, the source is assumed to be large enough such that all the incident rays corresponding to reflected rays hitting $\mathrm{D}$ originate within $\mathcal{S}$. Accordingly, the total irradiance at the detector center in this case reduces to

$$
E_{\mathrm{D}, \mathrm{LSSR}}^{b}(t)=\sum_{k, \ell} \frac{(m+1) \cos ^{m-1}\left(\theta_{\mathrm{R}_{k, \ell}^{\mathrm{S}}}\right) \cos \left(\theta_{\mathrm{R}_{k, \ell}, \mathrm{D}}^{b}\right)}{2 \pi A_{\mathrm{s}}\left\|\mathbf{R}_{\boldsymbol{k}, \ell} \mathbf{D}\right\|_{2}^{2}} B \cos \left(\theta_{\mathrm{R}_{k, \ell}}^{\mathrm{D}}\right) \iint_{\mathcal{R}_{k, \ell}^{b}} p\left(t-\tau\left(\mathrm{I}_{\mathrm{R}, \mathrm{D}}^{b}, \mathrm{R}, \mathrm{D}\right)\right) d A_{\mathrm{R}} .
$$


Hence, the impulse response can be expressed as

$$
h_{\mathrm{LSSR}}^{b}(t)=A_{\mathrm{PD}} \sum_{k, \ell} \frac{(m+1) \cos ^{m-1}\left(\theta_{\mathrm{R}_{k, \ell}}^{\mathrm{S}}\right) \cos \left(\theta_{\mathrm{R}_{k, \ell}, \mathrm{D}}^{b}\right)}{2 \pi A_{\mathrm{s}}\left\|\mathbf{R}_{\boldsymbol{k}, \ell} \mathbf{D}\right\|_{2}^{2}} B \cos \left(\theta_{\mathrm{R}_{k, \ell}}^{\mathrm{D}}\right) I_{b}(t),
$$

where

$$
I_{b}(t)=\iint_{\mathcal{R}_{k, \ell}^{b}} \delta\left(t-\tau\left(\mathrm{I}_{\mathrm{R}, \mathrm{D}}^{b}, \mathrm{R}, \mathrm{D}\right)\right) d A_{\mathrm{R}}
$$

The previous expression can be further simplified for negligible path delays for rays hitting the same reflecting element as

$$
h_{\mathrm{LSSR}}^{b}(t) \approx A_{\mathrm{PD}} \sum_{k, \ell} \frac{(m+1) \cos ^{m-1}\left(\theta_{\mathrm{R}_{k, \ell}^{\mathrm{S}}}\right) \cos \left(\theta_{\mathrm{R}_{k, \ell}, \mathrm{D}}^{b}\right)}{2 \pi A_{\mathrm{s}}\left\|\mathbf{R}_{k, \ell} \mathrm{D}\right\|_{2}^{2}} B \cos \left(\theta_{\mathrm{R}_{k, \ell}}^{\mathrm{D}}\right) A_{\mathrm{R}} \delta\left(t-\tau\left(\mathrm{S}, \mathrm{R}_{k, \ell}, \mathrm{D}\right)\right),
$$

where $A_{\mathrm{R}}$ represents the area of the reflecting element.

\section{TAPPED-DELAY LINE CHANNEL MODEL}

This section considers the temporal dispersive nature of the optical wireless channel and develops a tapped-delay line representation of the end-to-end channel. Towards this end, by following a similar procedure to that adopted in [28, Ch. 2], we express the received signal as a weighted sum of differently delayed replicas of the transmitted signal as

$y_{b}(t)=\sum_{a=0}^{L} q_{a}^{b} x\left(t-\frac{\ell}{B_{x}}\right)+n(t)$,

where $q_{a}^{b}$ represents the $a$-th tap coefficient and can be expressed as

$q_{a}^{b}=\left.h_{b}(t) * \operatorname{sinc}\left(B_{x} t\right)\right|_{t=\frac{a}{B_{x}}}$,

where ' $*$ ' represents the convolution operator. Using (5), (6), and evaluating the previous convolution, $q_{a}^{\mathrm{b}}$ can be expressed as

$$
q_{a}^{b}=\sum_{k, \ell} \iint_{\mathcal{R}_{k, \ell}^{b}} \frac{\rho_{b} A_{\mathrm{PD}} \operatorname{sinc}\left(a-B_{x} \tau\left(\mathrm{I}_{\mathrm{R}, \mathrm{P}}, \mathrm{R}, \mathrm{P}\right)\right)(m+1) \cos ^{m-1}\left(\theta_{\mathrm{R}, \mathrm{P}}^{\mathrm{I}_{\mathrm{R}}^{b}}\right)}{2 \pi A_{\mathrm{s}}\|\mathbf{R P}\|_{2}^{2}} \cos \left(\theta_{\mathrm{R}, \mathrm{P}}^{b}\right) \cos \left(\theta_{\mathrm{R}}^{\mathrm{P}}\right) B \mathbb{I}\left(\mathrm{I}_{\mathrm{R}, \mathrm{P}}^{b} \in \mathcal{S}\right) d A_{\mathrm{R}}
$$


where $x(t)$ is a band-limited signal with bandwidth $B_{x}, n(t)$ represents the noise term, $L \approx \tau_{\mathrm{d}} B_{x}$ is the number of significant taps, i.e., the number of resolvable paths, and $\tau_{\mathrm{d}}^{b}$ represents the channel delay spread. Consequently, (18) reduces to

$$
\begin{aligned}
q_{a}^{b} \leq \rho_{b} \sum_{k, \ell} & \frac{A_{\mathrm{PD}}(m+1) \cos ^{m-1}\left(\theta_{\mathrm{R}_{k, \ell}}^{\mathrm{S}}\right)}{2 \pi\left\|\mathbf{R}_{k, \ell} \mathrm{D}\right\|_{2}^{2}} \cos \left(\theta_{\mathrm{R}_{k, \ell}, \mathrm{D}}^{b}\right) \cos \left(\theta_{\mathrm{R}_{k, \ell}}^{\mathrm{D}}\right) \\
& \times\left. J\left(\frac{\mathrm{I}_{\mathrm{R}, \mathrm{D}, x}^{b}, \mathrm{I}_{\mathrm{R}, \mathrm{D}, y}^{b}}{\mathrm{R}_{x}, \mathrm{R}_{z}}\right)\right|_{\mathrm{R}=\mathrm{R}_{k, \ell}} \operatorname{sinc}\left(a-B_{x} \tau\left(\mathrm{I}_{\mathrm{R}, \mathrm{P}}, \mathrm{R}, \mathrm{P}\right)\right),
\end{aligned}
$$

for the point source case, and

$$
q_{a}^{b} \approx A_{\mathrm{PD}} \sum_{k, \ell} \frac{(m+1) \cos ^{m-1}\left(\theta_{\mathrm{R}_{k, \ell}}^{\mathrm{S}}\right) \cos \left(\theta_{\mathrm{R}_{k, \ell}, \mathrm{D}}\right)}{2 \pi A_{\mathrm{s}}\left\|\mathbf{R}_{k, \ell} \mathbf{D}\right\|_{2}^{2}} B \cos \left(\theta_{\mathrm{R}_{k, \ell}}^{\mathrm{D}}\right) A_{\mathrm{R}} \operatorname{sinc}\left(a-B_{x} \tau\left(\mathrm{I}_{\mathrm{R}, \mathrm{P}}, \mathrm{R}, \mathrm{P}\right)\right),
$$

for the large source small reflector case.

In this work, we define the delay spread as the difference between the maximum and the minimum delays encountered by the rays exiting the source and hitting the detector center, $\tau_{\max }^{b}, \tau_{\min }^{b}$, respectively. Hence, the delay spread is written as

$$
\tau_{\mathrm{d}}^{b}=\tau_{\max }^{b}-\tau_{\min }^{b}
$$

where $\tau_{\max }^{b}$ is found from,

$$
\begin{aligned}
\tau_{\max }^{b}=\max _{\mathrm{R}} & \tau\left(\mathrm{I}_{\mathrm{R}, \mathrm{D}}^{b}, \mathrm{R}, \mathrm{D}\right) \\
& \text { subject to } \mathrm{R} \in \bigcup_{k, \ell} \mathcal{R}_{k, \ell}, \mathrm{I}_{\mathrm{R}, \mathrm{D}}^{b} \in \mathcal{S},
\end{aligned}
$$

that can be rewritten equivalently as,

$$
\begin{array}{rll}
\tau_{\max }^{b}=\max _{k, \ell} & \max _{\mathrm{R}} & \tau\left(\mathrm{I}_{\mathrm{R}, \mathrm{D}}^{b}, \mathrm{R}, \mathrm{D}\right) \\
& \text { subject to } \quad \mathrm{R} \in \mathcal{R}_{k, \ell}, \mathrm{I}_{\mathrm{R}, \mathrm{D}}^{b} \in \mathcal{S},
\end{array}
$$

while $\tau_{\text {min }}^{b}$ is given by,

$$
\begin{aligned}
\tau_{\min }^{b}=\min _{\mathrm{R}} & \tau\left(\mathrm{I}_{\mathrm{R}, \mathrm{D}}^{b}, \mathrm{R}, \mathrm{D}\right) \\
& \text { subject to } \mathrm{R} \in \bigcup_{k, \ell} \mathcal{R}_{k, \ell}, \mathrm{I}_{\mathrm{R}, \mathrm{D}}^{b} \in \mathcal{S},
\end{aligned}
$$


or equivalently from,

$$
\begin{array}{rll}
\tau_{\min }^{b}=\min _{k, \ell} & \min _{\mathrm{R}} & \tau\left(\mathrm{I}_{\mathrm{R}, \mathrm{D}}^{b}, \mathrm{R}, \mathrm{D}\right) \\
& \text { subject to } & \mathrm{R} \in \mathcal{R}_{k, \ell}, \mathrm{I}_{\mathrm{R}, \mathrm{D}}^{b} \in \mathcal{S} .
\end{array}
$$

By expressing the second constraint in the previous optimization problems in terms of $\mathrm{R}_{x}$ and $\mathrm{R}_{z}$ based on [8, Eqn. (58), (59)] for the mirror array reflector, and their counter parts for the metasurface reflector, the complexity of the feasibility region becomes self evident. Consequently, we derive upper-bounds for the delay spread of both reflector types, the asymptotic case with very large number of reflecting elements, and the point source.

\section{A. Upper bound on the delay spread}

The fundamental idea behind the upper bounds derived in this section and the next section is based on considering all the rays exiting the source and hitting the IRS as contributors to the delay spread. This can be formulated as

$$
\tau_{\mathrm{d}, \mathrm{UB}}^{b}=\tau_{\max , \mathrm{UB}}^{b}-\tau_{\min , \mathrm{LB}}^{b}
$$

where

$$
\begin{array}{lll}
\tau_{\max , \mathrm{UB}}^{b}= & \max _{\mathrm{R}, \overline{\mathrm{I}}} & \tau(\overline{\mathrm{I}}, \mathrm{R}, \mathrm{D}) \\
& \text { subject to } & \mathrm{R} \in \bigcup_{k, \ell} \mathcal{R}_{k, \ell}, \overline{\mathrm{I}} \in \mathcal{S}, \\
\tau_{\text {min,LB }}^{b}= & \min _{\mathrm{R}, \overline{\mathrm{I}}} & \tau(\overline{\mathrm{I}}, \mathrm{R}, \mathrm{D}) \\
& \text { subject to } & \mathrm{R} \in \bigcup_{k, \ell} \mathcal{R}_{k, \ell}, \overline{\mathrm{I}} \in \mathcal{S},
\end{array}
$$

1) Mirror array reflector: To further simplify the computational complexity for the mirror array setup, we relax the locality constraint of the reflection point to be within the tightest bounding box for the mirror array setup. Consequently, the delay spread upper bound for the mirror array setup can be formulated as

$\tau_{\mathrm{d}, \mathrm{UB}}^{\mathrm{Mi}}=\tau_{\max , \mathrm{UB}}^{\mathrm{Mi}}-\tau_{\mathrm{min}, \mathrm{LB}}^{\mathrm{Mi}}$,

where

$$
\begin{aligned}
\tau_{\max , \mathrm{UB}}^{\mathrm{Mi}}= & \max _{\mathrm{R}, \overline{\mathrm{I}}} \tau(\overline{\mathrm{I}}, \mathrm{R}, \mathrm{D}) \\
& \text { subject to } \quad \mathrm{R} \in \mathcal{B}, \overline{\mathrm{I}} \in \mathcal{S},
\end{aligned}
$$


where $\mathcal{B}=\left\{\boldsymbol{A}: x_{\mathrm{L}} \leq A_{x} \leq x_{\mathrm{U}}, y_{\mathrm{L}} \leq A_{y} \leq y_{\mathrm{U}}\right.$, and $\left.z_{\mathrm{L}} \leq A_{z} \leq z_{\mathrm{U}}\right\}$ and $\mathcal{S}=\left\{\boldsymbol{A}: x_{\text {min }}^{\mathrm{s}} \leq\right.$ $A_{x} \leq x_{\max }^{\mathrm{s}}, y_{\min }^{\mathrm{s}} \leq A_{y} \leq y_{\max }^{\mathrm{s}}$, and $\left.z_{\text {min }}^{\mathrm{s}} \leq A_{z} \leq z_{\text {max }}^{\mathrm{s}}\right\}$, such that

$x_{\mathrm{L}}=\min _{\mathrm{R} \in \cup_{k, \ell} \mathcal{R}_{k, \ell}^{c}} \mathrm{R}_{x}, x_{\mathrm{U}}=\max _{\mathrm{R} \in \bigcup_{k, \ell} \mathcal{R}_{k, \ell}^{c}} \mathrm{R}_{x}$,

$y_{\mathrm{L}}=\min _{\mathrm{R} \in \cup_{k, \ell} \mathcal{R}_{k, \ell}^{c}} \mathrm{R}_{y}, y_{\mathrm{U}}=\max _{\mathrm{R} \in \bigcup_{k, \ell} \mathcal{R}_{k, \ell}^{c}} \mathrm{R}_{y}$,

$z_{\mathrm{L}}=\min _{\mathrm{R} \in \bigcup_{k, \ell} \mathcal{R}_{k, \ell}^{c}} \mathrm{R}_{z}, z_{\mathrm{U}}=\max _{\mathrm{R} \in \bigcup_{k, \ell} \mathcal{R}_{k, \ell}^{c}} \mathrm{R}_{z}$,

$\mathcal{R}_{k, \ell}^{\mathrm{c}}$ represents the set of four corner points of $\mathcal{R}_{k, \ell}$, and

$\tau_{\min , \mathrm{LB}}^{\mathrm{Mi}}=\min _{\mathrm{R}, \overline{\mathrm{I}}} \quad \tau(\overline{\mathrm{I}}, \mathrm{R}, \mathrm{D})$

subject to $\mathrm{R} \in \mathcal{B}, \overline{\mathrm{I}} \in \mathcal{S}$.

It can be seen clearly in (30) and (34) that the constraint qualification condition is satisfied. Hence, the necessity of the Karush-Kuhn-Tucker (KKT) conditions is guaranteed [29, Ch. 13]. The Lagrangian of (30), (34) can be expressed as

$$
\begin{aligned}
& \mathcal{L}=\sqrt{\left(h_{\mathrm{d}}-\mathrm{R}_{z}\right)^{2}+\left(x_{\mathrm{d}}-\mathrm{R}_{x}\right)^{2}+\left(y_{\mathrm{d}}-\mathrm{R}_{y}\right)^{2}}+\sqrt{\left(\overline{\mathrm{I}}_{x}-\mathrm{R}_{x}\right)^{2}+\mathrm{R}_{z}^{2}+\left(\overline{\mathrm{I}}_{y}-\mathrm{R}_{y}\right)^{2}} \\
& +\nu_{1}\left(\mathrm{R}_{x}-x_{\mathrm{L}}\right)+\mu_{1}\left(\mathrm{R}_{z}-z_{\mathrm{L}}\right)+\nu_{2}\left(\mathrm{R}_{x}-x_{\mathrm{U}}\right)+\mu_{2}\left(\mathrm{R}_{z}-z_{\mathrm{U}}\right)+\kappa_{1}\left(\overline{\mathrm{I}}_{x}-x_{\text {min }}^{\mathrm{s}}\right) \\
& +\zeta_{1}\left(\mathrm{R}_{y}-y_{\mathrm{L}}\right)+\zeta_{2}\left(\mathrm{R}_{y}-y_{\mathrm{U}}\right)+\kappa_{2}\left(\overline{\mathrm{I}}_{x}-x_{\text {max }}^{\mathrm{s}}\right)+o_{1}\left(\overline{\mathrm{I}}_{y}-y_{\text {min }}^{\mathrm{s}}\right)+o_{2}\left(\overline{\mathrm{I}}_{y}-y_{\max }^{\mathrm{s}}\right),
\end{aligned}
$$

where $\overline{\mathrm{I}}_{x}, \overline{\mathrm{I}}_{y}$ represent the $x$ - and $y$ - coordinates of $\overline{\mathrm{I}}$, Hence, the KKT stationarity conditions can be expressed as:

$$
\begin{aligned}
& \frac{\partial \mathcal{L}}{\partial \mathrm{R}_{x}}=0 \Longrightarrow \frac{\mathrm{R}_{x}-x_{\mathrm{d}}}{\ell_{\mathrm{RD}}}+\frac{\mathrm{R}_{x}-\overline{\mathrm{I}}_{x}}{\ell_{\mathrm{R} \overline{\mathrm{I}}}}+\nu_{1}+\nu_{2}=0 . \\
& \frac{\partial \mathcal{L}}{\partial \mathrm{R}_{y}}=0 \Longrightarrow \frac{\mathrm{R}_{y}-y_{\mathrm{d}}}{\ell_{\mathrm{RD}}}+\frac{\mathrm{R}_{y}-\overline{\mathrm{I}}_{y}}{\ell_{\mathrm{R} \overline{\mathrm{I}}}}+\kappa_{1}+\kappa_{2}=0, \\
& \frac{\partial \mathcal{L}}{\partial \mathrm{R}_{z}}=0 \Longrightarrow \frac{\mathrm{R}_{z}-h_{\mathrm{d}}}{\ell_{\mathrm{RD}}}+\frac{\mathrm{R}_{z}}{\ell_{\mathrm{R} \overline{\mathrm{I}}}}+\mu_{1}+\mu_{2}=0, \\
& \frac{\partial \mathcal{L}}{\partial \overline{\mathrm{I}}_{x}}=0 \Longrightarrow \frac{\overline{\mathrm{I}}_{x}-\mathrm{R}_{x}}{\ell_{\mathrm{R} \overline{\mathrm{I}}}}+\kappa_{1}+\kappa_{2}=0, \\
& \frac{\partial \mathcal{L}}{\partial \overline{\mathrm{I}}_{y}}=0 \Longrightarrow \frac{\overline{\mathrm{I}}_{y}-\mathrm{R}_{y}}{\ell_{\mathrm{R} \overline{\mathrm{I}}}}+o_{1}+o_{2}=0,
\end{aligned}
$$

where $\ell_{\mathrm{RD}}=\sqrt{\left(h_{\mathrm{d}}-\mathrm{R}_{z}\right)^{2}+\left(x_{\mathrm{d}}-\mathrm{R}_{x}\right)^{2}+\left(y_{\mathrm{d}}-\mathrm{R}_{y}\right)^{2}}$, and $\ell_{\mathrm{R} \overline{\mathrm{I}}}=\sqrt{\left(\overline{\mathrm{I}}_{x}-\mathrm{R}_{x}\right)^{2}+\mathrm{R}_{z}^{2}+\overline{\mathrm{I}}_{y}^{2}}$.

We assume that $y_{\min }^{\mathrm{s}}>y_{\mathrm{U}}$, hence, the optimal value for $\overline{\mathrm{I}}_{y}\left(\overline{\mathrm{I}}_{y}^{*}\right)$ for (30) and (34), respectively, is 
$y_{\max }^{\mathrm{s}}$ and $y_{\min }^{\mathrm{s}}$. By considering the different possibilities of the Lagrange multipliers associated with $\mathrm{R}_{x}, \mathrm{R}_{y}, \mathrm{R}_{z}$, and $\overline{\mathrm{I}}_{x}$ variables due to complementary slackness, the KKT stationary points of interest belong to one of the following cases:

TABLE I: KKT stationary points where $y_{\mathrm{L}}<\mathrm{R}_{y}^{*}<y_{\mathrm{U}}, x_{\min }^{\mathrm{s}}<\overline{\mathrm{I}}_{x}^{*}<x_{\max }^{\mathrm{s}}$,

\begin{tabular}{|c|c|c|c|}
\hline Case 1 & $\begin{aligned} x_{\mathrm{L}} & <\mathrm{R}_{x}^{*}<x_{\mathrm{U}} \\
z_{\mathrm{L}} & <\mathrm{R}_{z}^{*}<z_{\mathrm{U}}\end{aligned}$ & Not a KKT point & \\
\hline Case 2 & $\begin{array}{c}x_{\mathrm{L}}<\mathrm{R}_{x}^{*}<x_{\mathrm{U}} \\
\mathrm{R}_{z}^{*}=\mathrm{R}_{z}^{\mathrm{c}} \in\left\{z_{\mathrm{L}}, z_{\mathrm{U}}\right\}\end{array}$ & $\begin{array}{l}\overline{\mathrm{I}}_{x}^{*}=\mathrm{R}_{x}^{*}=x_{\mathrm{d}} \\
\mathrm{R}_{y, 1}^{*}=\mathrm{R}_{z}^{\mathrm{c}}\left(y_{\mathrm{d}}-\overline{\mathrm{I}}_{y}\right) / h_{\mathrm{d}}+\overline{\mathrm{I}}_{y} \\
\mathrm{R}_{y, 2}^{*}=h_{\mathrm{d}} \overline{\mathrm{I}}_{y}-\mathrm{R}_{z}^{\mathrm{c}}\left(y_{\mathrm{d}}+\overline{\mathrm{I}}_{y}\right) /\left(h_{\mathrm{d}}-2 \mathrm{R}_{z}^{\mathrm{c}}\right)\end{array}$ & $\begin{array}{l}(41) \\
(42) \\
(43)\end{array}$ \\
\hline Case 3 & $\begin{array}{c}\mathrm{R}_{x}^{*}=\mathrm{R}_{x}^{\mathrm{c}} \in\left\{x_{\mathrm{L}}, x_{\mathrm{U}}\right\} \\
z_{\mathrm{L}}<\mathrm{R}_{z}^{*}<z_{\mathrm{U}}\end{array}$ & Not a KKT point & \\
\hline Case 4 & $\begin{aligned} & \mathrm{R}_{x}^{*}=\mathrm{R}_{x}^{\mathrm{c}} \\
& \mathrm{R}_{z}^{*}=\mathrm{R}_{z}^{\mathrm{c}} \in\left\{x_{\mathrm{L}}, x_{\mathrm{U}}\right\} \\
&\left.z_{\mathrm{L}}, z_{\mathrm{U}}\right\}\end{aligned}$ & $\begin{array}{l}\overline{\mathrm{I}}_{x}^{*}=\mathrm{R}_{x}^{\mathrm{c}}, \\
\mathrm{R}_{y, 1}^{*}=\frac{-\Delta_{1}+\overline{\mathrm{I}}_{y}\left(\left(x_{\mathrm{d}}-\mathrm{R}_{x}^{\mathrm{c}}\right)^{2}+h_{\mathrm{d}}^{2}\right)+\left(\mathrm{R}_{z}^{\mathrm{c}}\right)^{2}\left(\overline{\mathrm{I}}_{y}-y_{\mathrm{d}}\right)-2 h_{\mathrm{d}} \mathrm{R}_{z}^{\mathrm{c}} \overline{\mathrm{I}}_{y}}{\left(x_{\mathrm{d}}-\mathrm{R}_{x}^{\mathrm{c}}\right)^{2}+h_{\mathrm{d}}\left(h_{\mathrm{d}}-2 \mathrm{R}_{z}^{\mathrm{c}}\right)}, \\
\mathrm{R}_{y, 2}^{*}=\frac{\Delta_{1}+\overline{\mathrm{I}}_{y}\left(\left(x_{\mathrm{d}}-\mathrm{R}_{x}^{\mathrm{c}}\right)^{2}+h_{\mathrm{d}}^{2}\right)+\left(\mathrm{R}_{z}^{\mathrm{c}}\right)^{2}\left(\overline{\mathrm{I}}_{y}-y_{\mathrm{d}}\right)-2 h_{\mathrm{d}} \mathrm{R}_{z}^{\mathrm{c}} \overline{\mathrm{I}}_{y}}{\left(x_{\mathrm{d}}-\mathrm{R}_{x}^{\mathrm{c}}\right)^{2}+h_{\mathrm{d}}\left(h_{\mathrm{d}}-2 \mathrm{R}_{z}^{\mathrm{c}}\right)} \\
\Delta_{1}=\sqrt{\left(\mathrm{R}_{z}^{\mathrm{c}}\right)^{2}\left(y_{\mathrm{d}}-\overline{\mathrm{I}}_{y}\right)^{2}\left(\left(x_{\mathrm{d}}-\mathrm{R}_{x}^{\mathrm{c}}\right)^{2}+\left(h_{\mathrm{d}}-\mathrm{R}_{z}^{\mathrm{c}}\right)^{2}\right)}\end{array}$ & $\begin{array}{l}(44) \\
(45) \\
(46)\end{array}$ \\
\hline
\end{tabular}

TABLE II: KKT stationary points where $y_{\mathrm{L}}<\mathrm{R}_{y}^{*}<y_{\mathrm{U}}, \overline{\mathrm{I}}_{x}^{*}=\overline{\mathrm{I}}_{x}^{\mathrm{c}} \in\left\{x_{\min }^{\mathrm{s}}, x_{\max }^{\mathrm{s}}\right\}$,

\begin{tabular}{|c|c|c|c|}
\hline Case 5 & $\begin{aligned} x_{\mathrm{L}} & <\mathrm{R}_{x}^{*}<x_{\mathrm{U}} \\
z_{\mathrm{L}} & <\mathrm{R}_{z}^{*}<z_{\mathrm{U}}\end{aligned}$ & Not a KKT point & \\
\hline Case 6 & $\begin{array}{c}x_{\mathrm{L}}<\mathrm{R}_{x}^{*}<x_{\mathrm{U}} \\
\mathrm{R}_{z}^{*}=\mathrm{R}_{z}^{\mathrm{c}} \in\left\{z_{\mathrm{L}}, z_{\mathrm{U}}\right\}\end{array}$ & $\begin{array}{l}\mathrm{R}_{x, 1}^{*}=\frac{\mathrm{R}_{z}^{\mathrm{c}}\left(x_{d}-\overline{\mathrm{I}}_{x}^{\mathrm{c}}\right)}{h_{\mathrm{d}}}+\overline{\mathrm{I}}_{x}^{\mathrm{c}}, \\
\mathrm{R}_{x, 2}^{*}=\frac{h_{\mathrm{d}} \overline{\mathrm{I}}_{x}^{\mathrm{c}}-\mathrm{R}_{z}^{\mathrm{c}}\left(x_{d}+\overline{\mathrm{I}}_{x}^{\mathrm{c}}\right)}{h_{\mathrm{d}}-2 \mathrm{R}_{z}^{\mathrm{c}}}, \\
\mathrm{R}_{y}^{*}=\frac{y_{\mathrm{d}}\left(\mathrm{R}_{x}^{*}-\overline{\mathrm{I}}_{x}^{\mathrm{c}}\right)+\overline{\mathrm{I}}_{y}\left(x_{d}-\mathrm{R}_{x}^{*}\right)}{x_{d}-\overline{\mathrm{I}}_{x}^{\mathrm{c}}} .\end{array}$ & $\begin{array}{l}(47) \\
(48) \\
(49)\end{array}$ \\
\hline Case 7 & $\begin{array}{c}\mathrm{R}_{x}^{*}=\mathrm{R}_{x}^{\mathrm{c}} \in\left\{x_{\mathrm{L}}, x_{\mathrm{U}}\right\} \\
z_{\mathrm{L}}<\mathrm{R}_{z}^{*}<z_{\mathrm{U}}\end{array}$ & $\begin{array}{l}\mathrm{R}_{y, 1}^{*}=\frac{y_{\mathrm{d}} \mathrm{R}_{x}^{\mathrm{c}}-y_{\mathrm{d}} \overline{\mathrm{I}}_{x}^{\mathrm{c}}+x_{d} \overline{\mathrm{I}}_{y}-\mathrm{R}_{x}^{\mathrm{c}} \overline{\mathrm{I}}_{y}}{x_{d}-\overline{\mathrm{I}}_{x}^{\mathrm{c}}} \\
\mathrm{R}_{y, 2}^{*}=\frac{-y_{\mathrm{d}} \mathrm{R}_{x}^{\mathrm{c}}+y_{\mathrm{d}} \overline{\mathrm{I}}_{x}^{\mathrm{c}}+x_{d} \overline{\mathrm{I}}_{y}-\mathrm{R}_{x}^{\mathrm{c}} \overline{\mathrm{I}}_{y}}{x_{d}-2 \mathrm{R}_{x}^{\mathrm{c}}+\overline{\mathrm{I}}_{x}^{\mathrm{c}}} \\
\mathrm{R}_{z}^{*}=\frac{h_{\mathrm{d}}\left(\mathrm{R}_{y}^{*}-\overline{\mathrm{I}}_{y}\right)}{y_{\mathrm{d}}-\overline{\mathrm{I}}_{y}}\end{array}$ & $\begin{array}{l}(50) \\
(51) \\
(52)\end{array}$ \\
\hline Case 8 & 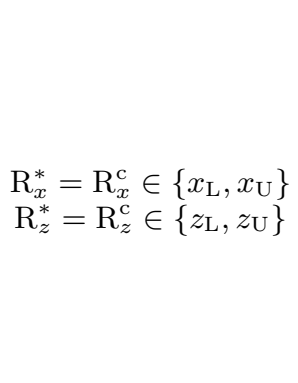 & $\begin{array}{l}\mathrm{R}_{y, 1}^{*}=\frac{\Delta_{2}+\overline{\mathrm{I}}_{y}\left(\left(x_{d}-\mathrm{R}_{x}^{\mathrm{c}}\right)^{2}+\left(h_{\mathrm{d}}-\left(\mathrm{R}_{z}^{\mathrm{c}}\right)\right)^{2}\right)+y_{\mathrm{d}}\left(-\left(\mathrm{R}_{x}^{\mathrm{c}}-\overline{\mathrm{I}}_{x}^{\mathrm{c}}\right)^{2}-\left(\mathrm{R}_{z}^{\mathrm{c}}\right)^{2}\right)}{\left(x_{d}-\overline{\mathrm{I}}_{x}^{\mathrm{c}}\right)\left(x_{d}-2 \mathrm{R}_{x}^{\mathrm{c}}+\overline{\mathrm{I}}_{x}^{\mathrm{c}}\right)+h_{\mathrm{d}}\left(h_{\mathrm{d}}-2 \mathrm{R}_{z}^{\mathrm{c}}\right)} \\
\mathrm{R}_{y, 2}^{*}=\frac{-\Delta_{2}+\overline{\mathrm{I}}_{y}\left(\left(x_{d}-\mathrm{R}_{x}^{\mathrm{c}}\right)^{2}+\left(h_{\mathrm{d}}-\left(\mathrm{R}_{z}^{\mathrm{c}}\right)\right)^{2}\right)+y_{\mathrm{d}}\left(-\left(\mathrm{R}_{x}^{\mathrm{c}}-\overline{\mathrm{I}}_{x}^{\mathrm{c}}\right)^{2}-\left(\mathrm{R}_{z}^{\mathrm{c}}\right)^{2}\right.}{\left(x_{d}-\overline{\mathrm{I}}_{x}^{\mathrm{c}}\right)\left(x_{d}-2 \mathrm{R}_{x}^{\mathrm{c}}+\overline{\mathrm{I}}_{x}^{\mathrm{c}}\right)+h_{\mathrm{d}}\left(h_{\mathrm{d}}-2 \mathrm{R}_{z}^{\mathrm{c}}\right)} \\
\Delta_{2}=\sqrt{\left(y_{\mathrm{d}}-\overline{\mathrm{I}}_{y}\right)^{2}\left(\left(x_{d}-\mathrm{R}_{x}^{\mathrm{c}}\right)^{2}+\left(h_{\mathrm{d}}-\left(\mathrm{R}_{z}^{\mathrm{c}}\right)\right)^{2}\right)\left(\left(\mathrm{R}_{x}^{\mathrm{c}}-\overline{\mathrm{I}}_{x}^{\mathrm{c}}\right)^{2}+\left(\mathrm{R}_{z}^{\mathrm{c}}\right)^{2}\right) .}\end{array}$ & $\begin{array}{l}\text { (53) } \\
\frac{2}{2} \\
(54)\end{array}$ \\
\hline
\end{tabular}


TABLE III: KKT stationary points where $\mathrm{R}_{y}^{*}=\mathrm{R}_{y}^{\mathrm{c}} \in\left\{y_{\mathrm{L}}, y_{\mathrm{U}}\right\}, x_{\min }^{\mathrm{s}}<\overline{\mathrm{I}}_{x}^{*}<x_{\max }^{\mathrm{s}}$,

\begin{tabular}{|c|c|c|c|}
\hline Case 9 & $\begin{aligned} x_{\mathrm{L}} & <\mathrm{R}_{x}^{*}<x_{\mathrm{U}} \\
z_{\mathrm{L}} & <\mathrm{R}_{z}^{*}<z_{\mathrm{U}}\end{aligned}$ & $\begin{array}{l}\overline{\mathrm{I}}_{x}^{*}=\mathrm{R}_{x}^{*}=x_{\mathrm{d}}, \\
\mathrm{R}_{z, 1}^{*}=\frac{h_{\mathrm{d}} \mathrm{R}_{y}^{\mathrm{c}}-h_{\mathrm{d}} \overline{\mathrm{I}}_{y}+y_{\mathrm{d}} z_{\mathrm{s}}-\mathrm{R}_{y}^{\mathrm{c}} z_{\mathrm{s}}}{y_{\mathrm{d}}-\overline{\mathrm{I}}_{y}}, \\
\mathrm{R}_{z, 2}^{*}=\frac{h_{\mathrm{d}} \overline{\mathrm{I}}_{y}-h_{\mathrm{d}} \mathrm{R}_{y}^{\mathrm{c}}}{y_{\mathrm{d}}-2 \mathrm{R}_{y}^{\mathrm{c}}+\overline{\mathrm{I}}_{y}}\end{array}$ & $\begin{array}{l}(55) \\
(56) \\
(57)\end{array}$ \\
\hline Case 10 & $\begin{array}{c}x_{\mathrm{L}}<\mathrm{R}_{x}^{*}<x_{\mathrm{U}} \\
\mathrm{R}_{z}^{*}=\mathrm{R}_{z}^{\mathrm{c}} \in\left\{z_{\mathrm{L}}, z_{\mathrm{U}}\right\}\end{array}$ & $\overline{\mathrm{I}}_{x}^{*}=\mathrm{R}_{x}^{*}=x_{\mathrm{d}}$ & (58) \\
\hline Case 11 & $\begin{array}{c}\mathrm{R}_{x}^{*}=\mathrm{R}_{x}^{\mathrm{c}} \in\left\{x_{\mathrm{L}}, x_{\mathrm{U}}\right\} \\
z_{\mathrm{L}}<\mathrm{R}_{z}^{*}<z_{\mathrm{U}}\end{array}$ & $\begin{array}{l}\overline{\mathrm{I}}_{x}^{*}=\mathrm{R}_{x}^{\mathrm{c}} \\
\mathrm{R}_{z, 1}^{*}=\frac{-\Delta_{3}+z_{\mathrm{s}}\left(\left(x_{d}-\mathrm{R}_{x}^{\mathrm{c}}\right)^{2}+y_{\mathrm{d}}^{2}\right)-h_{\mathrm{d}}\left(\mathrm{R}_{y}^{\mathrm{c}}-\overline{\mathrm{I}}_{y}\right)^{2}+\mathrm{R}_{y}^{\mathrm{c}} z_{\mathrm{s}}\left(\mathrm{R}_{y}^{\mathrm{c}}-2 y_{\mathrm{d}}\right)}{\left(y_{\mathrm{d}}-\overline{\mathrm{I}}_{y}\right)\left(y_{\mathrm{d}}-2 \mathrm{R}_{y}^{\mathrm{c}}+\overline{\mathrm{I}}_{y}\right)+\left(x_{d}-\mathrm{R}_{x}^{\mathrm{c}}\right)^{2}} \\
\mathrm{R}_{z, 2}^{*}=\frac{\Delta_{3}+z_{\mathrm{s}}\left(\left(x_{d}-\mathrm{R}_{x}^{\mathrm{c}}\right)^{2}+y_{\mathrm{d}}^{2}\right)-h_{\mathrm{d}}\left(\mathrm{R}_{y}^{\mathrm{c}}-\overline{\mathrm{I}}_{y}\right)^{2}+\mathrm{R}_{y}^{\mathrm{c}} z_{\mathrm{s}}\left(\mathrm{R}_{y}^{\mathrm{c}}-2 y_{\mathrm{d}}\right)}{\left(y_{\mathrm{d}}-\overline{\mathrm{I}}_{y}\right)\left(y_{\mathrm{d}}-2 \mathrm{R}_{y}^{\mathrm{c}}+\overline{\mathrm{I}}_{y}\right)+\left(x_{d}-\mathrm{R}_{x}^{\mathrm{c}}\right)^{2}} \\
\Delta_{3}=\sqrt{\left(h_{\mathrm{d}}-z_{\mathrm{s}}\right)^{2}\left(\mathrm{R}_{y}^{\mathrm{c}}-\overline{\mathrm{I}}_{y}\right)^{2}\left(\left(x_{d}-\mathrm{R}_{x}^{\mathrm{c}}\right)^{2}+\left(y_{\mathrm{d}}-\mathrm{R}_{y}^{\mathrm{c}}\right)^{2}\right)}\end{array}$ & $\begin{array}{l}(59) \\
(60) \\
(61)\end{array}$ \\
\hline Case 12 & $\begin{aligned} \mathrm{R}_{x}^{*} & =\mathrm{R}_{x}^{\mathrm{c}} \in\left\{x_{\mathrm{L}}, x_{\mathrm{U}}\right\} \\
\mathrm{R}_{z}^{*} & =\mathrm{R}_{z}^{\mathrm{c}} \in\left\{z_{\mathrm{L}}, z_{\mathrm{U}}\right\}\end{aligned}$ & $\overline{\mathrm{I}}_{x}^{*}=\mathrm{R}_{x}^{\mathrm{c}}$ & (62) \\
\hline
\end{tabular}

TABLE IV: KKT stationary points where $\mathrm{R}_{y}^{*}=\mathrm{R}_{y}^{\mathrm{c}} \in\left\{y_{\mathrm{L}}, y_{\mathrm{U}}\right\}, \overline{\mathrm{I}}_{x}^{*}=\overline{\mathrm{I}}_{x}^{\mathrm{c}} \in\left\{x_{\min }^{\mathrm{s}}, x_{\max }^{\mathrm{s}}\right\}$

\begin{tabular}{|c|c|c|c|}
\hline Case 13 & $\begin{aligned} x_{\mathrm{L}} & <\mathrm{R}_{x}^{*}<x_{\mathrm{U}} \\
z_{\mathrm{L}} & <\mathrm{R}_{z}^{*}<z_{\mathrm{U}}\end{aligned}$ & $\begin{array}{l}\mathrm{R}_{z, 1}^{*}=\frac{h_{\mathrm{d}}\left(\mathrm{R}_{y}^{\mathrm{c}}-\overline{\mathrm{I}}_{y}\right)}{y_{\mathrm{d}}-\overline{\mathrm{I}}_{y}}, \\
\mathrm{R}_{z, 2}^{*}=\frac{h_{\mathrm{d}}\left(\overline{\mathrm{I}}_{y}-\mathrm{R}_{y}^{\mathrm{c}}\right)}{y_{\mathrm{d}}-2 \mathrm{R}_{y}^{\mathrm{c}}+\overline{\mathrm{I}}_{y}}, \\
\mathrm{R}_{x}^{*}=\frac{\overline{\mathrm{I}}_{x}\left(h_{\mathrm{d}}-\mathrm{R}_{z}^{*}\right)+x_{d} \mathrm{R}_{z}^{*}}{h_{\mathrm{d}}} .\end{array}$ & $\begin{array}{l}(63) \\
(64) \\
(65)\end{array}$ \\
\hline Case 14 & $\begin{array}{c}x_{\mathrm{L}}<\mathrm{R}_{x}^{*}<x_{\mathrm{U}} \\
\mathrm{R}_{z}^{*}=\mathrm{R}_{z}^{\mathrm{c}} \in\left\{z_{\mathrm{L}}, z_{\mathrm{U}}\right\}\end{array}$ & $\begin{array}{l}\mathrm{R}_{x, 1}^{*}=\frac{\Delta_{4}+\overline{\mathrm{I}}_{x}^{\mathrm{c}}\left(\left(y_{\mathrm{d}}-\mathrm{R}_{y}^{\mathrm{c}}\right)^{2}+\left(h_{\mathrm{d}}-\mathrm{R}_{z}^{\mathrm{c}}\right)^{2}\right)-x_{d}\left(\left(\mathrm{R}_{y}^{\mathrm{c}}-\overline{\mathrm{I}}_{y}\right)^{2}+\left(\mathrm{R}_{z}^{\mathrm{c}}\right)^{2}\right)}{\left(y_{\mathrm{d}}-\overline{\mathrm{I}}_{y}\right)\left(y_{\mathrm{d}}-2 \mathrm{R}_{y}^{\mathrm{c}}+\overline{\mathrm{I}}_{y}\right)+h_{\mathrm{d}}\left(h_{\mathrm{d}}-2 \mathrm{R}_{z}^{\mathrm{c}}\right)} \\
\mathrm{R}_{x, 2}^{*}=\frac{-\Delta_{4}+\overline{\mathrm{I}}_{x}^{\mathrm{c}}\left(\left(y_{\mathrm{d}}-\mathrm{R}_{y}^{\mathrm{c}}\right)^{2}+\left(h_{\mathrm{d}}-\mathrm{R}_{z}^{\mathrm{c}}\right)^{2}\right)-x_{d}\left(\left(\mathrm{R}_{y}^{\mathrm{c}}-\overline{\mathrm{I}}_{y}\right)^{2}+\left(\mathrm{R}_{z}^{\mathrm{c}}\right)^{2}\right)}{\left(y_{\mathrm{d}}-\overline{\mathrm{I}}_{y}\right)\left(y_{\mathrm{d}}-2 \mathrm{R}_{y}^{\mathrm{c}}+\overline{\mathrm{I}}_{y}\right)+h_{\mathrm{d}}\left(h_{\mathrm{d}}-2 \mathrm{R}_{z}^{\mathrm{c}}\right)} \\
\Delta_{4}=\sqrt{\left(x_{d}-\overline{\mathrm{I}}_{x}^{\mathrm{c}}\right)^{2}\left(\left(y_{\mathrm{d}}-\mathrm{R}_{y}^{\mathrm{c}}\right)^{2}+\left(h_{\mathrm{d}}-\mathrm{R}_{z}^{\mathrm{c}}\right)^{2}\right)\left(\left(\mathrm{R}_{y}^{\mathrm{c}}-\overline{\mathrm{I}}_{y}\right)^{2}+\left(\mathrm{R}_{z}^{\mathrm{c}}\right)^{2}\right)}\end{array}$ & $\begin{array}{l} \\
(66) \\
(67)\end{array}$ \\
\hline Case 15 & $\begin{array}{c}\mathrm{R}_{x}^{*}=\mathrm{R}_{x}^{\mathrm{c}} \in\left\{x_{\mathrm{L}}, x_{\mathrm{U}}\right\} \\
z_{\mathrm{L}}<\mathrm{R}_{z}^{*}<z_{\mathrm{U}}\end{array}$ & $\begin{aligned} \mathrm{R}_{z, 1}^{*} & =\frac{h_{\mathrm{d}}^{2}}{h_{\mathrm{d}}-\frac{\sqrt{h_{\mathrm{d}}^{2}\left(\left(x_{d}-\mathrm{R}_{x}^{\mathrm{c}}\right)^{2}+\left(y_{\mathrm{d}}-\mathrm{R}_{y}^{\mathrm{c}}\right)^{2}\right)\left(\left(\mathrm{R}_{x}^{\mathrm{c}}-\overline{\mathrm{I}}_{x}^{\mathrm{c}}\right)^{2}+\left(\mathrm{R}_{y}^{\mathrm{c}}-\overline{\mathrm{I}}_{y}\right)^{2}\right)}}{\left(\mathrm{R}_{x}^{\mathrm{c}}-\overline{\mathrm{I}}_{x}^{\mathrm{c}}\right)^{2}+\left(\mathrm{R}_{y}^{\mathrm{c}}-\overline{\mathrm{I}}_{y}\right)^{2}}} \\
\mathrm{R}_{z, 2}^{*}= & \frac{h_{\mathrm{d}}^{2}}{h_{\mathrm{d}}+\frac{\sqrt{h_{\mathrm{d}}^{2}\left(\left(x_{d}-\mathrm{R}_{x}^{\mathrm{c}}\right)^{2}+\left(y_{\mathrm{d}}-\mathrm{R}_{y}^{\mathrm{c}}\right)^{2}\right)\left(\left(\mathrm{R}_{x}^{\mathrm{c}}-\overline{\mathrm{I}}_{x}^{\mathrm{c}}\right)^{2}+\left(\mathrm{R}_{y}^{\mathrm{c}}-\overline{\mathrm{I}}_{y}\right)^{2}\right)}}{\left(\mathrm{R}_{x}^{\mathrm{c}}-\overline{\mathrm{I}}_{x}^{\mathrm{c}}\right)^{2}+\left(\mathrm{R}_{y}^{\mathrm{c}}-\overline{\mathrm{I}}_{y}\right)^{2}}} .\end{aligned}$ & (68) \\
\hline Case 16 & $\begin{aligned} \mathrm{R}_{x}^{*} & =\mathrm{R}_{x}^{\mathrm{c}} \in\left\{x_{\mathrm{L}}, x_{\mathrm{U}}\right\} \\
\mathrm{R}_{z}^{*} & =\mathrm{R}_{z}^{\mathrm{c}} \in\left\{z_{\mathrm{L}}, z_{\mathrm{U}}\right\}\end{aligned}$ & & \\
\hline
\end{tabular}


The optimal solution of (30), and (34), respectively, can be expressed as

$$
\begin{aligned}
& \left(\mathbf{R}_{\text {max }, \text { Mi }}^{*}, \overline{\mathbf{I}}_{\text {max }}^{*}\right)=\arg \max _{\left(\mathrm{R}^{*}, \overline{\mathrm{I}}^{*}\right) \in \bigcup_{n=1}^{16} \mathcal{P}_{n}} \tau(\overline{\mathrm{I}}, \mathrm{R}, \mathrm{D}), \\
& \left(\mathbf{R}_{\text {min,Mi }, M}^{*} \overline{\mathbf{I}}_{\text {min }}^{*}\right)=\arg \min _{\left(\mathrm{R}^{*}, \overline{\mathbf{I}}^{*}\right) \in \bigcup_{n=1}^{16} \mathcal{P}_{n}} \tau(\overline{\mathrm{I}}, \mathrm{R}, \mathrm{D}),
\end{aligned}
$$

where $\mathcal{P}_{n}$ represents the set of feasible stationary points satisfying the conditions of the $n-$ th case presented in the previous tables ${ }^{4}$.

2) Metasurface reflector: For the metasurface reflector, the delay spread upper bound can be computed directly using (26) based on (27) and (28). It can be noticed that (30) and (34) reduce to (27) and (28), respectively, for $\mathrm{R}_{y}=y_{\mathrm{L}}=y_{\mathrm{U}}=0$. Consequently, the optimal values of $\mathrm{R}_{x}$, $\mathrm{R}_{z}, \overline{\mathrm{I}}_{x}$, and $\overline{\mathrm{I}}_{y}$ can be computed as follows

$$
\begin{gathered}
\left(\mathbf{R}_{\text {max }, \mathrm{Ms}}^{*}, \overline{\mathbf{I}}_{\text {max }}^{*}\right)=\arg \max _{\left(\mathrm{R}^{*}, \overline{\mathrm{I}}^{*}\right) \in \bigcup_{9 \leq n \leq 16} \mathcal{P}_{n}} \tau(\overline{\mathrm{I}}, \mathrm{R}, \mathrm{D}), \\
\left(\mathbf{R}_{\text {min,Ms }}^{*}, \overline{\mathbf{I}}_{\text {min }}^{*}\right)=\arg \min _{\left(\mathrm{R}^{*}, \overline{\mathrm{I}}^{*}\right) \in \bigcup_{9 \leq n \leq 16} \mathcal{P}_{n}} \tau(\overline{\mathrm{I}}, \mathrm{R}, \mathrm{D}),
\end{gathered}
$$

with $\boldsymbol{e}_{\mathbf{2}}{ }^{\mathrm{T}} \overline{\mathbf{I}}_{\max }^{*}=y_{\max }^{\mathrm{s}}, \boldsymbol{e}_{\mathbf{2}}{ }^{\mathrm{T}} \overline{\mathbf{I}}_{\text {min }}^{*}=y_{\min }^{\mathrm{s}}$, and $\mathrm{R}_{y}^{\mathrm{c}}=0$.

\section{B. Asymptotic delay spread}

In this section, we derive the asymptotic delay spread for both reflector types as $n_{\mathrm{m}}$ and $n_{\mathrm{p}}$ increase unboundedly. It can be noticed that in such case, all the rays reaching the detector center from both reflector types, originate at the source center. On the other hand, all the rays coming from other points on the source will not reach the detector center by the virtue of the uniqueness of reflection direction imposed by the generalized law of reflection. Consequently, the asymptotic delay spread can be expressed as

$$
\tau_{\mathrm{d}}^{\text {Asym }}=\lim _{n_{b} \rightarrow \infty} \tau_{\mathrm{d}}=\tau_{\max }^{\text {Asym }}-\tau_{\min }^{\text {Asym }},
$$

where $n_{b} \in\left\{n_{\mathrm{p}}, n_{\mathrm{m}}\right\}$,

$$
\begin{gathered}
\tau_{\max }^{\mathrm{Asym}}=\max _{\mathrm{R} \in \bigcup_{k, \ell} \mathcal{R}_{k, \ell}} \tau(\mathrm{S}, \mathrm{R}, \mathrm{D}), \\
\tau_{\min }^{\mathrm{Asym}}=\min _{\mathrm{R} \in \bigcup_{k, \ell} \mathcal{R}_{k, \ell}} \tau(\mathrm{S}, \mathrm{R}, \mathrm{D}) .
\end{gathered}
$$


TABLE V: Default simulation parameters.

\begin{tabular}{|c|c|c|c|c|}
\hline$z_{\mathrm{s}}=1 \mathrm{~m}$ & $l_{\mathrm{s}}=10 \mathrm{~cm}$ & $w_{\mathrm{s}}=5 \mathrm{~cm}$ & $x_{\mathrm{s}}=-0.5 \mathrm{~m}$ & $y_{\mathrm{s}}=1 \mathrm{~m}$ \\
\hline$x_{\mathrm{d}}=-3 \mathrm{~m}$ & $y_{\mathrm{d}}=1 \mathrm{~m}$ & $h_{\mathrm{d}}=2.15 \mathrm{~m}$ & $l_{\mathrm{d}}=1 \mathrm{~cm}$ & $w_{\mathrm{d}}=1 \mathrm{~cm}$ \\
\hline$n_{\mathrm{p}}=10$ & $w_{\mathrm{p}}=10 \mathrm{~cm}$ & $h_{\mathrm{p}}=1.5 \mathrm{~cm}$ & $\Delta_{w_{\mathrm{p}}}=0 \mathrm{~cm}$ & $\Delta_{h_{\mathrm{p}}}=0 \mathrm{~cm}$ \\
\hline$n_{\mathrm{p}}=10$ & $w_{\mathrm{p}}=10 \mathrm{~cm}$ & $h_{\mathrm{p}}=1.5 \mathrm{~cm}$ & $\Delta_{w_{\mathrm{p}}}=0 \mathrm{~cm}$ & $\Delta_{h_{\mathrm{p}}}=0 \mathrm{~cm}$ \\
\hline$m=2$ & $\rho_{\mathrm{p}}=0.8$ & $\rho_{\mathrm{m}}=0.8$ & & \\
\hline
\end{tabular}

It can be seen that (75), and (76) are special cases of (27) and (28), respectively, when $\overline{\mathrm{I}} \rightarrow \mathrm{S}$, $y_{\mathrm{L}}=y_{\mathrm{U}}=\mathrm{R}_{y}^{\mathrm{c}}=0$. Consequently, the optimal solution of (75), and (76) can be expressed as

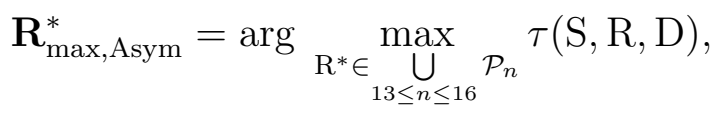

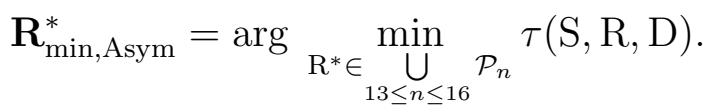

\section{Point source}

It is clear that when $A_{\mathrm{S}} \rightarrow 0$, only the chief rays originating at $\mathrm{S}$ and hitting the IRS at $\mathrm{R}_{k, \ell} \forall k, \ell$ will be reflected to $\mathrm{D}$ and, hence, contribute to the delay spread. Consequently, the point source delay spread can be expressed as

$$
\begin{aligned}
& \tau_{\mathrm{d}}^{\mathrm{PS}}=\lim _{A_{\mathrm{s}} \rightarrow 0} \tau_{\mathrm{d}}=\tau_{\max }^{\mathrm{PS}}-\tau_{\min }^{\mathrm{PS}}, \\
& \tau_{\max }^{\mathrm{PS}}=\max _{k, \ell} \quad \tau\left(\mathrm{S}, \mathrm{R}_{k, \ell}, \mathrm{D}\right), \\
& \tau_{\min }^{\mathrm{PS}}=\min _{k, \ell} \tau\left(\mathrm{S}, \mathrm{R}_{k, \ell}, \mathrm{D}\right) .
\end{aligned}
$$

It is worth mentioning that $\tau_{\mathrm{d}}^{\mathrm{PS}}$ represents a lower bound on $\tau_{\mathrm{d}}^{b}$ as $\tau_{\max }^{\mathrm{PS}} \leq \tau_{\max }^{b}$ and $\tau_{\min }^{\mathrm{PS}} \geq \tau_{\min }^{b}$ as (23), (24), (80), and (81) possess the same objective function and the feasibility region of (80) and (81) is a subset of the feasibility region of (23) and (24).

\section{Simulation Results}

In this section, we evaluate the temporal behavior of the IRS-based VLC channel and its susceptibility to changes in several system parameters. Towards this aim, we present the continuoustime impulse response for both reflectors and the equivalent tapped delay line model. In addition, we study the impact of the number of reflecting elements, the source area, the reflector size, the reflector aspect ratio and the detector location on the channel delay spread for both reflector 
types. The significance of these parameters on the power distribution in the detection plane and the received power has been highlighted in [8]. Hence, it is necessary to evaluate their impact on the delay spread performance metric and shed the light on the potential tradeoff between the two metrics. We assume the system parameter values provided in Table V; unless otherwise stated. The assumed source and detector specifications are inspired by their counterparts in [30], [31] and practical LED luminaires, while the relative geometric layout is chosen to set a general asymmetric configuration to avoid drawing conclusions based on special cases. Moreover, the reflector dimensions and number of elements were adjusted such that the reflected power is large enough to neglect the LoS received power if available at the detector and are inherited from [8]. In all the conducted simulations, the reflectors are assumed to be tightly packed, i.e., $\Delta_{w_{\mathrm{p}}}=\Delta_{w_{\mathrm{m}}}=\Delta_{h_{\mathrm{p}}}=\Delta_{h_{\mathrm{m}}}=0$, and the source center is horizontally aligned with both reflectors centers.

In the first simulation, we study the normalized impulse response of both reflectors $\left(\tilde{h}_{\mathrm{b}}(\tau), b \in\right.$ $\{\mathrm{MS}, \mathrm{Mi}\})$, where $\tilde{h}_{\mathrm{b}}(\tau)=h_{\mathrm{b}}(\tau) / \sqrt{\int_{0}^{\infty} h_{\mathrm{b}}^{2}(\tau) d \tau}$, for different reflector aspect ratio $\left(w_{\mathrm{b}} / h_{\mathrm{b}}\right)$ values. Throughout this simulation, the total compact area of both reflectors $A_{\mathrm{R}}=n_{\mathrm{b}} w_{\mathrm{b}} h_{\mathrm{b}}$ is kept $0.15 \mathrm{~m}^{2}$, where the reflector total compact width takes the values $\{0.387,1,2.5\} \mathrm{m}$, while the reflector height values are $\{0.387,0.15,0.06\} \mathrm{m}$. It can be seen in Fig. 4a and Fig. $4 \mathrm{~b}$, that the impulse response of both reflectors constitutes as a superposition of overlapping pulses representing the individual contributions of the metasurface patches and the mirror array reflecting elements. The non-uniform spacing between the received pulses owes to the nonuniform travel times of the rays reaching the detector from different reflectors. It can be seen clearly from Fig. $4 \mathrm{a}$ and $4 \mathrm{~b}$ that as the aspect ratio of the reflector increases, the received power at the detector is distributed over a longer time span. The null channel response between some of the successive pulses is attributed to the inactive portions of the reflecting elements when the reflecting element width increases.

In the second simulation, we monitor the normalized impulse response of both reflector types for different reflector area values. We assume the reflector compact width admits the following values $\{0.25,1,2.5\} \mathrm{m}$, whilst the compact reflector height is kept constant. It can be seen from Fig. 5a and Fig. 5b that, in agreement with the aspect ratio simulation, as the reflector area increases, the total received power is distributed over a larger time span. It can be further noticed that the pulses representing the individual contributions of the reflecting elements become wider and hence the number of nulls decreases as can be seen clearly in Fig. 5a for the $A_{\mathrm{R}}=0.375 \mathrm{~m}^{2}$ 


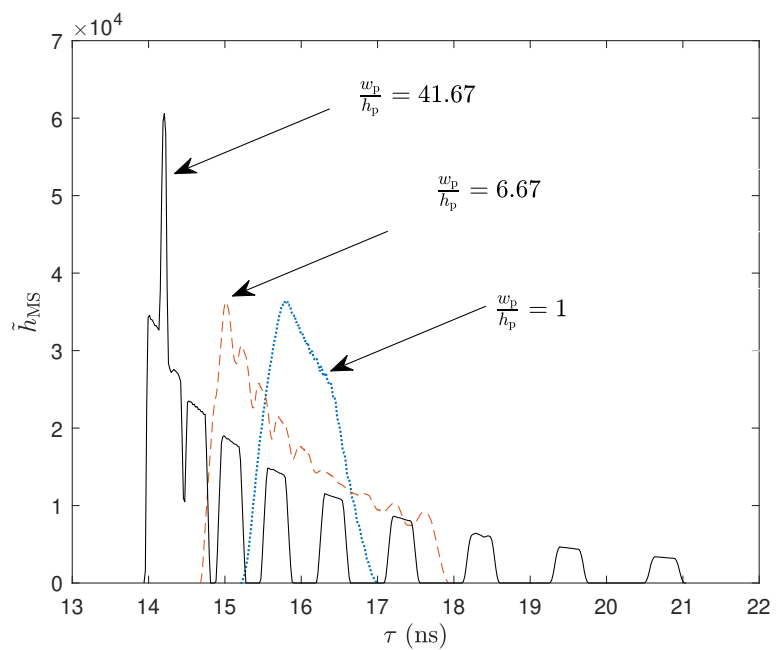

(a) Metasurface reflector

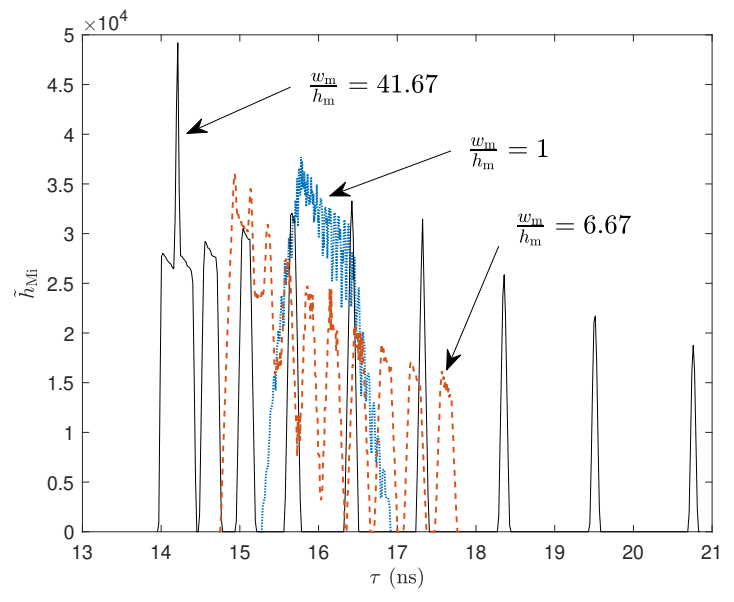

(b) Mirror Array reflector

Fig. 4: Normalized impulse response for different aspect ratios

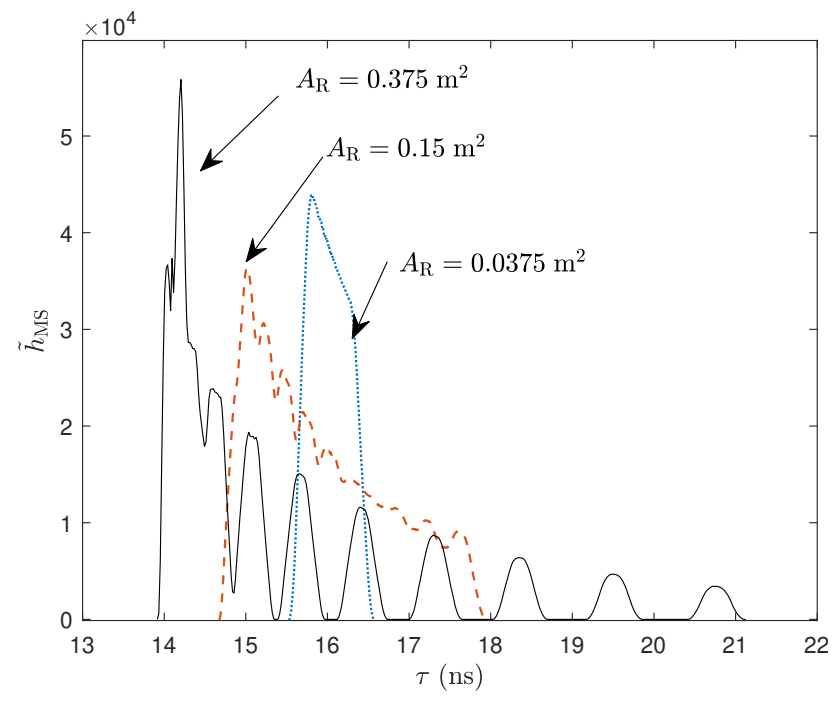

(a) Metasurface reflector

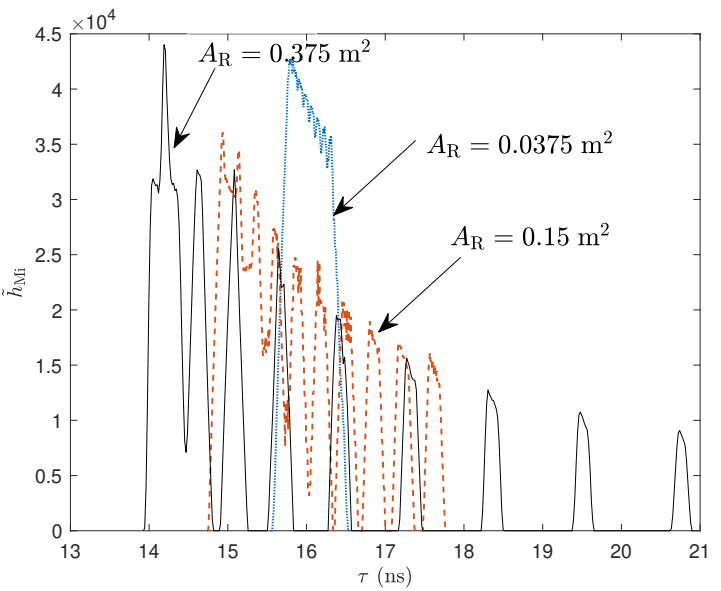

(b) Mirror Array reflector

Fig. 5: Normalized impulse response for different reflector total areas

reflector.

In Table VI and VII, we summarize the normalized tapped-delay line coefficients for the two previously mentioned simulations, where $\bar{q}_{a}=q_{a} / \sqrt{\sum_{k=0}^{6} q_{k}^{2}} \quad \forall a$.

In the third simulation, we evaluate the impact of the reflector area and the number of reflecting elements on the number of taps of the tapped-delay line model. Throughout this simulation, the total area of both reflectors is kept constant. It can be seen in Fig. 6a and Fig. 6b that $L_{b}$ is a non- 
TABLE VI: Tapped delay line model parameters for the metasurface reflector

\begin{tabular}{|c|c|c|c|c|c|c|c|c|}
\hline$w_{\mathrm{R}}(\mathrm{m})$ & $h_{\mathrm{R}}(\mathrm{m})$ & $\bar{q}_{0}$ & $\bar{q}_{1}$ & $\bar{q}_{2}$ & $\bar{q}_{3}$ & $\bar{q}_{4}$ & $\bar{q}_{5}$ & $\bar{q}_{6}$ \\
\hline 0.3873 & 0.3873 & 0.5346 & 0.8451 & 0 & 0 & 0 & 0 & 0 \\
\hline 1 & 0.15 & 0.4876 & 0.8277 & 0.2704 & 0.0637 & 0 & 0 & 0 \\
\hline 2.5 & 0.06 & 0.5986 & 0.7538 & 0.1488 & 0.1999 & 0.0483 & 0.0948 & -0.0039 \\
\hline 0.25 & 0.15 & 0.8410 & 0.5411 & 0 & 0 & 0 & 0 & 0 \\
\hline 1 & 0.15 & 0.4876 & 0.8277 & 0.2704 & 0.0637 & 0 & 0 & 0 \\
\hline 2.5 & 0.15 & 0.6204 & 0.7343 & 0.1512 & 0.2018 & 0.0434 & 0.1021 & -0.0051 \\
\hline
\end{tabular}

TABLE VII: Tapped delay line model parameters for mirror the array reflector

\begin{tabular}{|c|c|c|c|c|c|c|c|c|}
\hline$w_{\mathrm{R}}(\mathrm{m})$ & $h_{\mathrm{R}}(\mathrm{m})$ & $\bar{q}_{0}$ & $\bar{q}_{1}$ & $\bar{q}_{2}$ & $\bar{q}_{3}$ & $\bar{q}_{4}$ & $\bar{q}_{5}$ & $\bar{q}_{6}$ \\
\hline 0.3873 & 0.3873 & 0.57472 & 0.81835 & 0 & 0 & 0 & 0 & 0 \\
\hline 1 & 0.15 & 0.5427 & 0.80069 & 0.25371 & 0 & 0 & 0 & 0 \\
\hline 2.5 & 0.06 & 0.68531 & 0.68287 & 0.12293 & 0.19173 & 0.038994 & 0.10318 & 0 \\
\hline 0.25 & 0.15 & 0.85358 & 0.52096 & 0 & 0 & 0 & 0 & 0 \\
\hline 1 & 0.15 & 0.5427 & 0.80069 & 0.25371 & 0 & 0 & 0 & 0 \\
\hline 2.5 & 0.15 & 0.66735 & 0.6975 & 0.13277 & 0.19341 & 0.042121 & 0.10637 & -0.0045051 \\
\hline
\end{tabular}

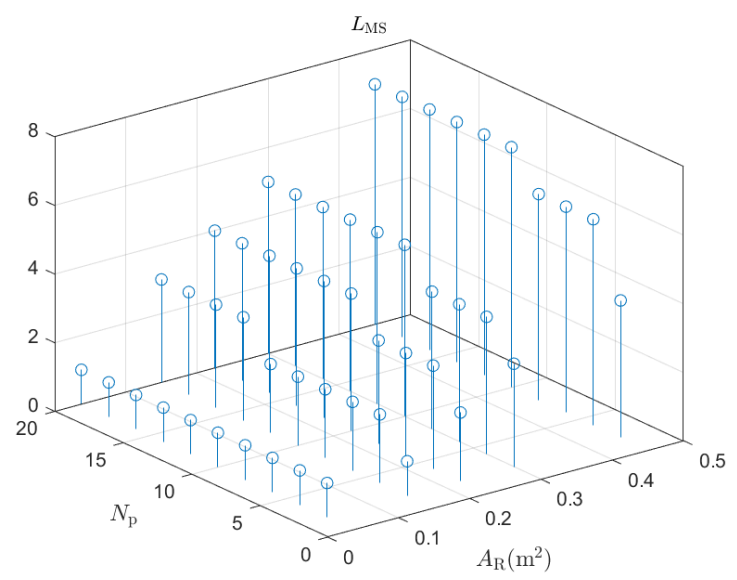

(a) Metasurface reflector

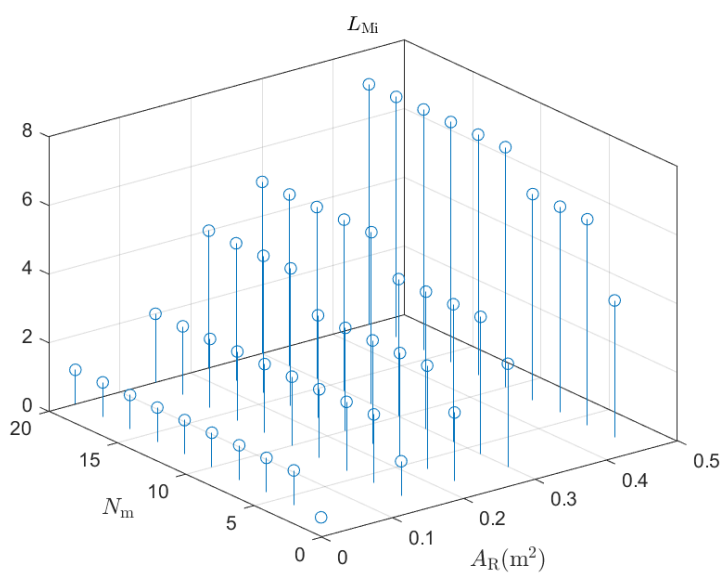

(b) Mirror Array reflector

Fig. 6: Tapped delay line filter order vs Reflector area and number of reflecting elements

decreasing function of the number of reflecting elements and the total reflector area. Nonetheless, the rate of increase of the function with $A_{\mathrm{R}}$ is more significant than the corresponding rate of change of the function with $N_{b}$. The observed performance owes to the underlying delay spread behavior with the number of reflecting elements and the total reflector area, as detailed in the following simulations.

In the fourth simulation, we investigate the behavior of the delay spread imposed by the two 


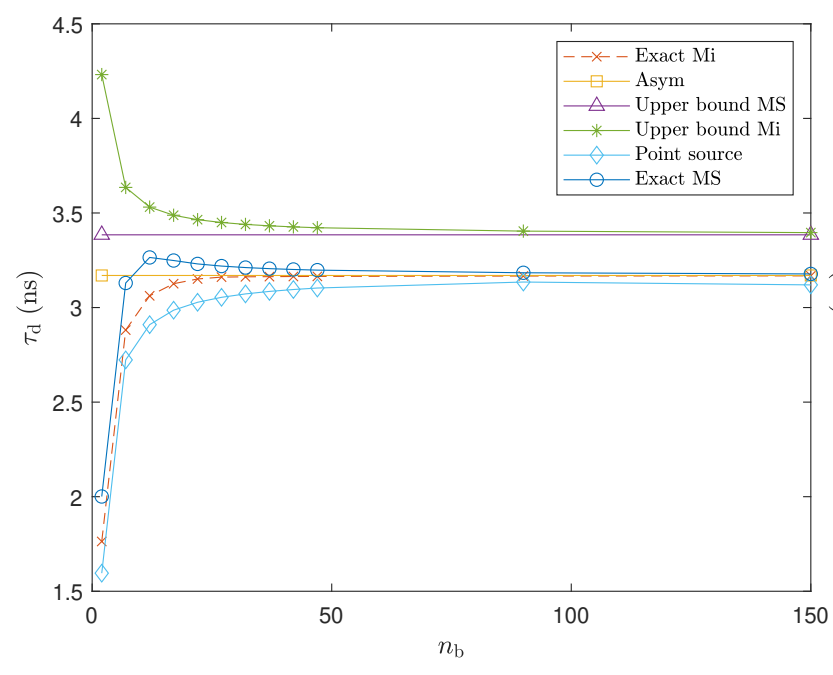

(a) $A_{\mathrm{R}}=0.15 \mathrm{~m}^{2}, A_{\mathrm{s}}=0.005 \mathrm{~m}^{2}$

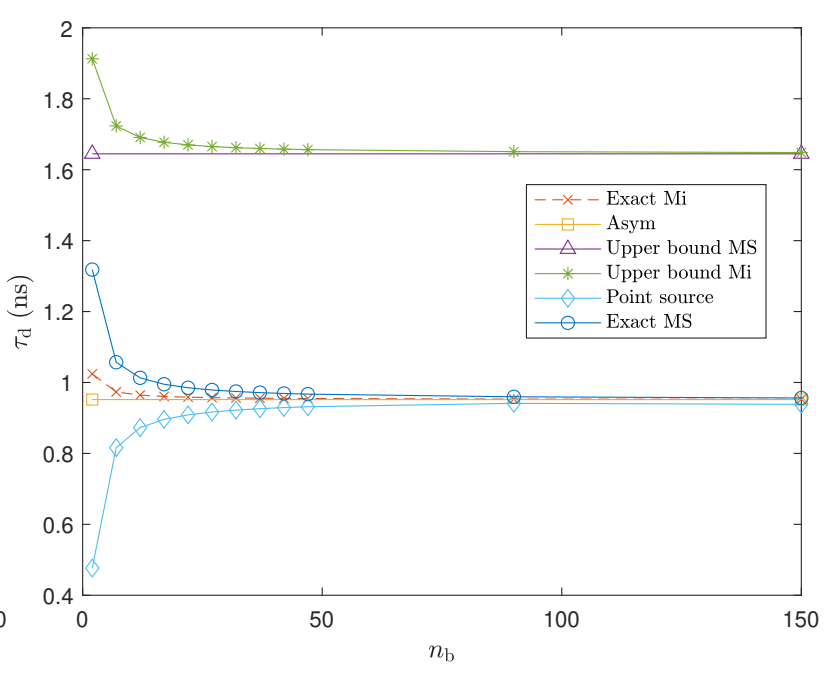

(b) $A_{\mathrm{R}}=0.0375 \mathrm{~m}^{2}, A_{\mathrm{s}}=0.125 \mathrm{~m}^{2}$

Fig. 7: Delay spread vs number of reflecting elements

reflector types versus the number of reflecting elements. In this regard, we plot $\tau_{\mathrm{d}}^{\mathrm{MS}}, \tau_{\mathrm{d}}^{\mathrm{Mi}}$, their corresponding upper bounds $\tau_{\mathrm{d}, \mathrm{UB}}^{\mathrm{MS}}, \tau_{\mathrm{d}, \mathrm{UB}}^{\mathrm{Mi}}$, and the point source delay spread, which acts as a lower bound for $\tau_{d}^{b}$ versus $n_{b}$. We consider two different cases for the relationship between the source area and the reflector area. It is important to recall that the delay spread is affected by two key factors: the active source area and the active reflector area, i.e., the set of points on the source and the reflector, respectively, contributing to the computed irradiance. It can be seen in Fig. 7a that as $n_{b}$ increases, while the total reflector area is kept constant, the delay spread exhibits, in general, a unimodal behavior. This owes to the increase of the total active reflector area which dominates the performance for small $n_{b}$ values, and the decrease of the active source area which dominates for large $n_{b}$ values.

Hence, the general behaviour of the overall delay spread for both reflectors is that it increases (chief ray delay variations dominate when the number of reflecting elements is small) till it reaches a maximum, then decreases when the delay spread is dominated by the local delay variations of rays reflected from the same element as can be seen in Fig. 7a, which finally settles at the asymptotic delay spread. It can be seen in Fig. $7 b$ that as the delay spread decreases with $n_{b}$ till it reaches $\tau_{\mathrm{d}}^{\text {Asym }}$. This is attributed to the decreasing source active area and the constant reflector active area.

In the fifth simulation, we study the impact of the source area on the delay spread for both reflector types. It can be seen from Fig. 8 that the delay spread increases till it saturates. The delay 


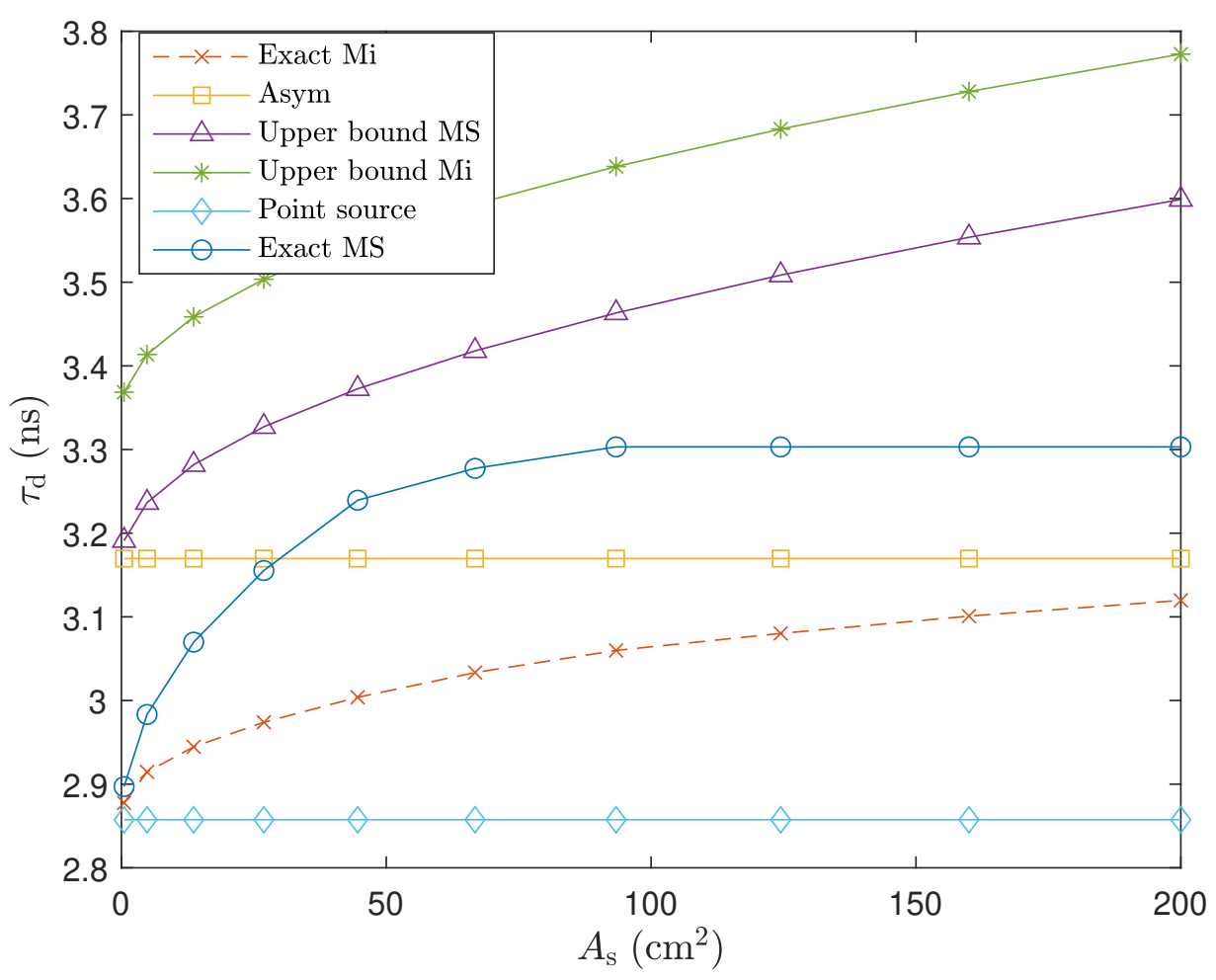

Fig. 8: Delay spread vs source area

spread performance owes to the increase of the active area of the individual reflecting elements. However, it is limited by the source area for small $A_{\mathrm{s}}$ values, where the active reflector area becomes the whole reflector area. In addition, it can be noticed that the gap between the delay spread upper bound and the delay spread increases as $A_{\mathrm{s}}$ increases, as they are controlled by the source and reflectors physical areas, not the active ones.

Finally, we study the effect of the detector location on the delay spread for both reflectors. It can be observed in Fig. 9 that as the detector moves further from the reflector, $r_{\mathrm{d}}$ increases, the delay spread decreases. This is explained by the significant similarity between the path lengths of all the rays traveling from the reflector to the detector center due to small reflection point location variation compared with $\overline{\mathrm{R}} \mathrm{D}$ for large $r_{\mathrm{d}}$ values.

\section{A. System performance evaluation}

This section uses the previously derived channel model to evaluate the two proposed reflectorbased systems performance. To this end, we assume an on-off keying transmission scheme with 


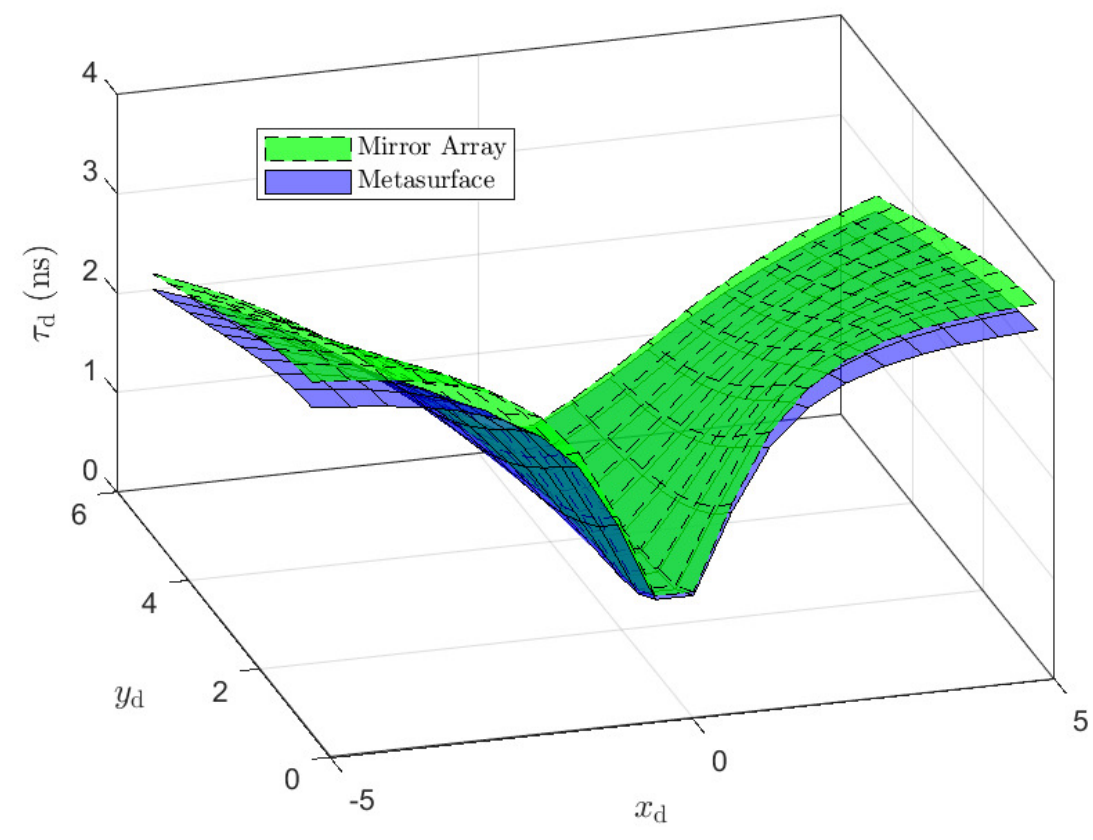

Fig. 9: Delay spread vs detector location

a signaling rate of $800 \mathrm{Mbps}$, and rectangular pulse shapes. We use a zero-forcing equalizer cascading the photodetector at the receiving end to combat the inter-symbol interference from the channel temporal dispersion. The receiver experiences random thermal noise modeled by an additive white Gaussian random process.

For benchmarking purposes, we compare the performance of the proposed system against a VLC system where we replace the IRS-based system with an array of generalized Lambertian point sources. We assume the locations of the point sources coincide with the centers of the reflecting elements and possess the same overall output power as the source. Moreover, we use a uniformly allocated power amongst the individual source array elements, and their Lambertian order is assumed to be 2, which is typical for LEDs. Furthermore, each element is oriented such that the line joining the source element and the detector center is perfectly aligned with the normal to that element.

In Fig. 10, we plot the bit error rate of both reflector-based systems for the scenario associated with the channel taps provided in the third row of Table VI and VII against the LoS signal to noise ratio. This scenario serves as a lower bound on the performance of the considered IRSaided systems associated with the first three rows of Table VI and VII (associated with the 


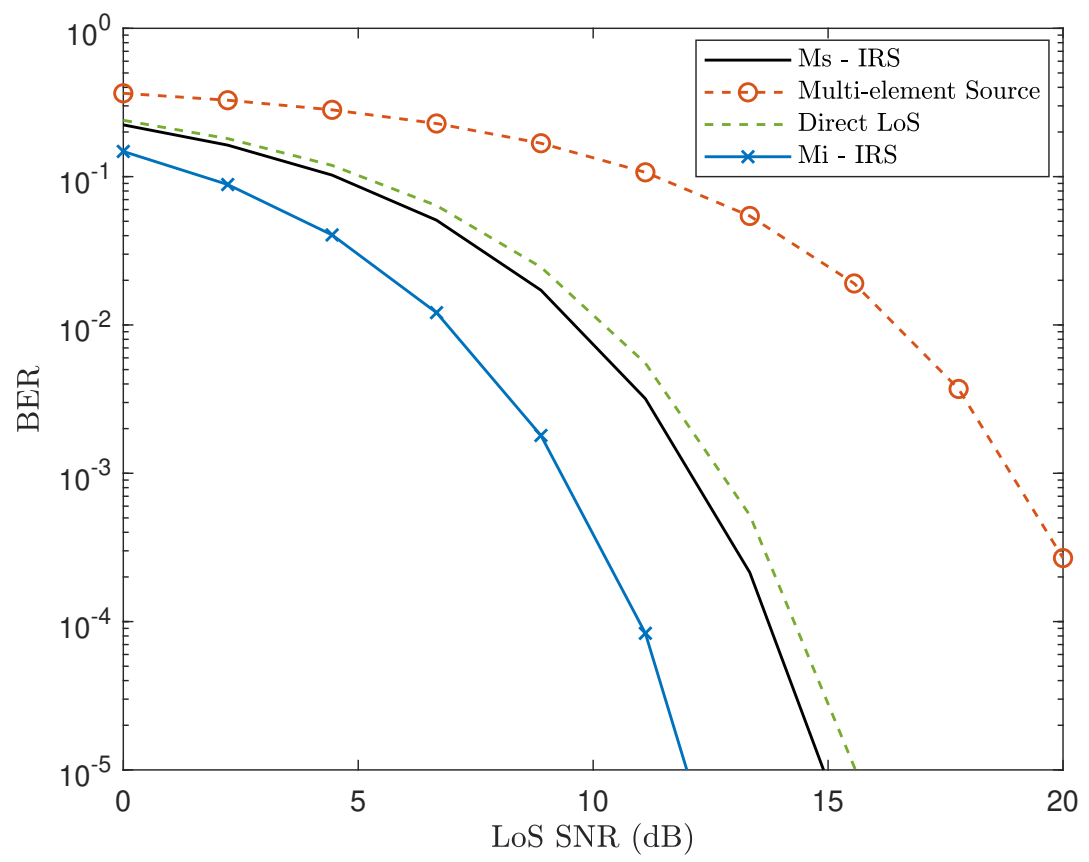

Fig. 10: Bit error rate vs LoS signal to noise ratio

aspect ratio simulation) due to the large delay spread imposed by such reflector geometry. It is clear that the mirror array IRS-based system outperforms its metasurface-based counterpart, owing to the less reflected beam spreading captured by the reflected beam intensity metric in [8, (78)] and [8, Fig. 10a]. In addition, both IRS-aided systems outperform the LoS, due to the signal gain they achieve as a result of their power focusing capability highlighted in [8, (80)] and [8, Fig. 9a,9b]. The performance degradation of the source array system is due to its low directivity abilities compared with the proposed IRS-based schemes, despite the close location to the receiver and the orientation alignment.

\section{CONCLUSION}

We characterized the temporal behavior of the metasurface and the mirror array IRS-aided VLC systems. Specifically, we derived the exact continuous-time impulse response for both reflector types. In addition, we provided simpler expressions for the point source and the large source small reflector schemes. Moreover, we presented the corresponding tapped-delay line model for the derived impulse response. Furthermore, we derived upper and lower bounds for the delay spread incurred for the two reflector types, in addition to the asymptotic delay spread when 
the number of reflecting elements increases significantly. Finally, we did extensive simulations to evaluate the system performance. It was found that the smaller the source area, the tighter the derived upper and lower bounds become. In addition, it was found that the delay spread experiences unimodal performance with a local maximum as the number of elements increases.

\section{APPENDIX A}

\section{EXCLUDED STATIONARY POINTS}

Case 1: $y_{\mathrm{L}}<\mathrm{R}_{y}^{*}<y_{\mathrm{U}}, x_{\min }^{\mathrm{s}}<\overline{\mathrm{I}}_{x}^{*}<x_{\max }^{\mathrm{s}}, x_{\mathrm{L}}<\mathrm{R}_{x}^{*}<x_{\mathrm{U}}, z_{\mathrm{L}}<\mathrm{R}_{z}^{*}<z_{\mathrm{U}}$

$$
\frac{\mathrm{R}_{z}-h_{\mathrm{d}}}{\sqrt{\left(h_{\mathrm{d}}-\mathrm{R}_{z}\right)^{2}\left(\frac{\left(y_{\mathrm{d}}-\overline{\mathrm{I}}_{y}\right)^{2}}{\left(h_{\mathrm{d}}-z_{\mathrm{s}}\right)^{2}}+1\right)}}+\frac{\mathrm{R}_{z}-z_{\mathrm{s}}}{\sqrt{\left(\mathrm{R}_{z}-z_{\mathrm{s}}\right)^{2}\left(\frac{\left(y_{\mathrm{d}}-\overline{\mathrm{I}}_{y}\right)^{2}}{\left(h_{\mathrm{d}}-z_{\mathrm{s}}\right)^{2}}+1\right)}}=0 .
$$

The previous equation holds only if $\mathrm{R}_{z}^{*}>h_{\mathrm{d}}$ or $\mathrm{R}_{z}^{*}<0$ which contradicts the assumptions. Hence, this case does not represent a KKT point.

Case 3: $y_{\mathrm{L}}<\mathrm{R}_{y}^{*}<y_{\mathrm{U}}, x_{\min }^{\mathrm{s}}<\overline{\mathrm{I}}_{x}^{*}<x_{\max }^{\mathrm{s}}, \mathrm{R}_{x}^{*}=\mathrm{R}_{x}^{\mathrm{c}} \in\left\{x_{\mathrm{L}}, x_{\mathrm{U}}\right\}, z_{\mathrm{L}}<\mathrm{R}_{z}^{*}<z_{\mathrm{U}}$

$$
\frac{\mathrm{R}_{z}-h_{\mathrm{d}}}{\sqrt{\frac{\left(h_{\mathrm{d}}-\mathrm{R}_{z}\right)^{2}\left(y_{\mathrm{d}}-\overline{\mathrm{I}}_{y}\right)^{2}}{h_{\mathrm{d}}{ }^{2}}+\left(x_{\mathrm{d}}-\mathrm{R}_{x}\right)^{2}+\left(h_{\mathrm{d}}-\mathrm{R}_{z}\right)^{2}}}+\frac{\mathrm{R}_{z}}{\sqrt{\frac{\left(y_{\mathrm{d}}-\overline{\mathrm{I}}_{y}\right)^{2}\left(\mathrm{R}_{z}\right)^{2}}{h_{\mathrm{d}}^{2}}+\left(\mathrm{R}_{x}-\overline{\mathrm{I}}_{x}\right)^{2}+\left(\mathrm{R}_{z}\right)^{2}}}=0 .
$$

For this equation to hold, $\mathrm{R}_{x}^{*}=x_{\mathrm{d}}$ which violates the assumptions. Consequently this is not a KKT point.

Case 5: $y_{\mathrm{L}}<\mathrm{R}_{y}^{*}<y_{\mathrm{U}}, \overline{\mathrm{I}}_{x}^{*} \in\left\{x_{\min }^{\mathrm{s}}, x_{\max }^{\mathrm{s}}\right\}, x_{\mathrm{L}}<\mathrm{R}_{x}^{*}<x_{\mathrm{U}}, z_{\mathrm{L}}<\mathrm{R}_{z}^{*}<z_{\mathrm{U}}$

$$
\frac{\mathrm{R}_{z}-h_{\mathrm{d}}}{\sqrt{\frac{\left(h_{\mathrm{d}}-\mathrm{R}_{z}\right)^{2}\left(\left(x_{d}-\overline{\mathrm{I}}_{x}\right)^{2}+\left(y_{\mathrm{d}}-\overline{\mathrm{I}}_{y}\right)^{2}+\left(h_{\mathrm{d}}-z_{\mathrm{s}}\right)^{2}\right)}{\left(h_{\mathrm{d}}-z_{\mathrm{s}}\right)^{2}}}}+\frac{\mathrm{R}_{z}-z_{\mathrm{s}}}{\sqrt{\frac{\left(\mathrm{R}_{z}-z_{\mathrm{s}}\right)^{2}\left(\left(x_{d}-\overline{\mathrm{I}}_{x}\right)^{2}+\left(y_{\mathrm{d}}-\overline{\mathrm{I}}_{y}\right)^{2}+\left(h_{\mathrm{d}}-z_{\mathrm{s}}\right)^{2}\right)}{\left(h_{\mathrm{d}}-z_{\mathrm{s}}\right)^{2}}}}=0 .
$$

This equation does not have a feasible solution. Hence, it does not represent a KKT point.

\section{REFERENCES}

[1] M. A. Khalighi and M. Uysal, "Survey on free space optical communication: A communication theory perspective," IEEE Commun. Surveys Tuts., vol. 16, no. 4, pp. 2231-2258, Jun. 2014.

[2] S. Dang, O. Amin, B. Shihada, and M.-S. Alouini, "What should 6G be?" Nature Electronics, vol. 3, no. 1, pp. 20-29, 2020. [Online]. Available: https://doi.org/10.1038/s41928-019-0355-6

[3] W. Saad, M. Bennis, and M. Chen, "A vision of 6G wireless systems: Applications, trends, technologies, and open research problems," IEEE network, 2019.

[4] A. M. Abdelhady, O. Amin, A. Chaaban, B. Shihada, and M. Alouini, "Downlink resource allocation for dynamic TDMAbased VLC systems," IEEE Transactions on Wireless Communications, vol. 18, no. 1, pp. 108-120, Jan 2019. 
[5] — - "Spectral-efficiency—illumination pareto front for energy harvesting enabled VLC systems," IEEE Transactions on Communications, vol. 67, no. 12, pp. 8557-8572, Dec 2019.

[6] A. M. Abdelhady, O. Amin, B. Shihada, and M. Alouini, "Spectral efficiency and energy harvesting in multi-cell SLIPT systems," IEEE Transactions on Wireless Communications, pp. 1-1, 2020.

[7] M. S. Rea, The IESNA lighting handbook: reference \& application. IESNA, 2000.

[8] A. M. Abdelhady, A. K. Salem, O. Amin, B. Shihada, and M.-S. Alouini, "Visible light communications via intelligent reflecting surfaces: Metasurfaces vs mirror arrays," IEEE Open J. Commun. Soc., vol. 2, pp. 1-20, 2021.

[9] M. Najafi and R. Schober, "Intelligent reflecting surfaces for free space optical communications," in 2019 IEEE Global Communications Conference (GLOBECOM). IEEE, 2019, pp. 1-7.

[10] M. Najafi, B. Schmauss, and R. Schober, "Intelligent reflecting surfaces for free space optical communications," arXiv preprint arXiv:2005.04499v2, 2021.

[11] H. Ajam, M. Najafi, V. Jamali, and R. Schober, "Channel modeling for IRS-assisted FSO systems," in 2021 IEEE Wireless Communications and Networking Conference (WCNC). Nanjing: IEEE, May 2021, pp. 1-7.

[12] C. Valagiannopoulos, T. A. Tsiftsis, and V. Kovanis, "Metasurface-enabled interference mitigation in visible light communication architectures," Journal of Optics, vol. 21, no. 11, p. 115702, Oct 2019.

[13] A. R. Ndjiongue, T. Ngatched, O. A. Dobre, and H. Haas, "Re-configurable intelligent surface-based VLC receivers using tunable liquid-crystals: The concept," arXiv preprint arXiv:2101.02369.

[14] H. Wang, Z. Zhang, B. Zhu, J. Dang, and L. Wu, “Two new approaches to optical IRSs: Schemes and comparative analysis," arXiv preprint arXiv:2012.15398, 2020.

[15] J. M. Kahn and J. R. Barry, "Wireless infrared communications,” Proc. IEEE, vol. 85, no. 2, pp. 265-298, Feb. 1997.

[16] F. R. Gfeller and U. Bapst, "Wireless in-house data communication via diffuse infrared radiation," Proceedings of the IEEE, vol. 67, no. 11, pp. 1474-1486, 1979.

[17] R. T. Valadas and A. de Oliveira Duarte, "Sectored receivers for indoor wireless optical communication systems," in 5th IEEE Int. Symp. on Personal, Indoor and Mobile Radio Commun., Wireless Networks-Catching the Mobile Future., The Hague, Sep. 1994, pp. 1090-1095.

[18] J. B. Carruthers and J. M. Kahn, "Modeling of nondirected wireless infrared channels," IEEE transactions on communications, vol. 45, no. 10, pp. 1260-1268, 1997.

[19] S. Karp, R. M. Gagliardi, S. E. Moran, and L. B. Stotts, Optical channels: fibers, clouds, water, and the atmosphere. Springer Science \& Business Media, 2013.

[20] Z. Ghassemlooy, W. Popoola, and S. Rajbhandari, Optical wireless communications: system and channel modelling with Matlabß. CRC press, 2019.

[21] J. Chen and C. Yan, "A channel model for indoor visible light communication system with specular reflection," in 2017 16th International Conference on Optical Communications and Networks (ICOCN). IEEE, Aug. 2017, pp. 1-3.

[22] J. Rufo, J. Rabadan, V. Guerra, and R. Perez-Jimenez, "BRDF models for the impulse response estimation in indoor optical wireless channels," IEEE Photonics Technology Letters, vol. 29, no. 17, pp. 1431-1434, 2017.

[23] F. Miramirkhani and M. Uysal, "Channel modeling and characterization for visible light communications," IEEE Photonics Journal, vol. 7, no. 6, pp. 1-16, 2015.

[24] A. Street, P. Stavrinou, D. O’brien, and D. Edwards, "Indoor optical wireless systems-a review," Optical and Quantum Electronics, vol. 29, no. 3, pp. 349-378, 1997.

[25] R. Protection and I.-I. Protection, "Guidelines on limits of exposure for broad-band incoherent optical radiation (0.38 to 3 нm)," Health Phys, vol. 73, no. 3, pp. 539-597, 1997. 
[26] D. H. Sliney and J. Mellerio, Safety with lasers and other optical sources: a comprehensive handbook. Springer Science \& Business Media, 2013.

[27] M. Born and E. Wolf, "Principles of optics: electromagnetic theory of propagation, interference and diffraction of light," 1999.

[28] D. Tse and P. Viswanath, Fundamentals of wireless communication. Cambridge university press, 2005.

[29] K. Wainwright et al., Fundamental methods of mathematical economics/Alpha C. Chiang, Kevin Wainwright. Boston, Mass.: McGraw-Hill/Irwin, 2005.

[30] T. Komine and M. Nakagawa, "Fundamental analysis for visible-light communication system using LED lights," IEEE transactions on Consumer Electronics, vol. 50, no. 1, pp. 100-107, 2004.

[31] S. Dimitrov and H. Haas, Principles of LED light communications: towards networked Li-Fi. Cambridge University Press, 2015. 